\title{
Defining locality as a problem difficulty measure in genetic programming
}

\author{
Edgar Galván-López • James McDermott • \\ Michael O’Neill • Anthony Brabazon
}

Received: 25 August 2010/Revised: 28 February 2011/Published online: 2 April 2011

(C) Springer Science+Business Media, LLC 2011

\begin{abstract}
A mapping is local if it preserves neighbourhood. In Evolutionary Computation, locality is generally described as the property that neighbouring genotypes correspond to neighbouring phenotypes. A representation has high locality if most genotypic neighbours are mapped to phenotypic neighbours. Locality is seen as a key element in performing effective evolutionary search. It is believed that a representation that has high locality will perform better in evolutionary search and the contrary is true for a representation that has low locality. When locality was introduced, it was the genotype-phenotype mapping in bitstringbased Genetic Algorithms which was of interest; more recently, it has also been used to study the same mapping in Grammatical Evolution. To our knowledge, there are few explicit studies of locality in Genetic Programming (GP). The goal of this paper is to shed some light on locality in GP and use it as an indicator of problem difficulty. Strictly speaking, in GP the genotype and the phenotype are not distinct. We attempt to extend the standard quantitative definition of genotype-phenotype locality to the genotype-fitness mapping by considering three possible definitions. We consider the effects of these definitions in both continuous- and discrete-valued fitness functions. We compare three different GP representations (two of them induced by using different function sets and the other using a slightly different GP encoding) and six different mutation operators. Results indicate that one definition of locality is better in predicting performance.
\end{abstract}

E. Galván-López $(\bowtie) \cdot J$. McDermott · M. O’Neill · A. Brabazon

Natural Computing Research and Applications Group, University College Dublin, Dublin, Ireland e-mail: edgar.galvan@ucd.ie

J. McDermott

e-mail: james.m.mcdermott@ucd.ie

M. O’Neill

e-mail: m.oneill@ucd.ie

A. Brabazon

e-mail: anthony.brabazon@ucd.ie 
Keywords Locality - Genotype-phenotype mapping - Genotype-fitness mapping · Problem hardness - Genetic programming

\section{Introduction}

The concept of a fitness landscape [69] has dominated the way geneticists think about biological evolution and has been adopted within the Evolutionary Computation (EC) community and many others as a way to visualise evolution dynamics. Over the years, researchers have defined fitness landscapes in slightly different ways (e.g., [30, 36] and [62]). All of them have in common the use of three main elements: search space $x$, neighbourhood mapping $\chi$ and fitness function $f$. More formally, a fitness landscape, as specified in [58], is normally defined as a triplet $(x, \chi, f)$ : (a) a set $x$ of configurations, (b) a notion $\chi$ of neighbourhood, distance or accessibility on $x$, and finally, (c) a fitness function $f$.

How an algorithm explores and exploits a landscape is a key element of evolutionary search. Rothlauf $[53,56]$ has described and analysed the importance of locality in performing effective evolutionary search of landscapes. In EC, locality refers to how well neighbouring genotypes correspond to neighbouring phenotypes, and is useful as an indicator of problem difficulty. Similarly, the principle of strong causality states that for successful search, a small change in genotype should result in a small change in fitness [2]. In other words, the design process of an algorithm should be guided by the locality principle [50].

In his research, Rothlauf distinguished two forms of locality, low and high. A representation has high locality if most neighbouring genotypes correspond to neighbouring phenotypes, that is small genotypic changes result in small phenotypic changes. On the other hand, a representation has low locality if many neighbouring genotypes do not correspond to neighbouring phenotypes. It is demonstrated that a representation of high locality is necessary for efficient evolutionary search. No threshold between low and high locality is established in Rothlauf's work, nor indeed in ours. Instead, we study locality as a relative concept: higher locality is assumed to lead to improved performance. In Sect. 2 we give quantitative definitions of locality allowing the relative locality of different representations and operators to be compared. Also, in Sect. 2, we re-label these two concepts with the goal to avoid misinterpretations.

In his original studies, Rothlauf used GAs with bitstrings to conduct his experiments [52] (and more recently he further used the idea of locality to study Grammatical Evolution at the genotype level [56]). To our knowledge, there are few explicit studies on locality using the typical Genetic Programming (GP) [33, 46] representation (i.e., tree-like structures).

The goal of this paper then is to shed some light on the degree of locality present in GP. That is,

- We extend the notion of genotype-phenotype locality to the genotype-fitness case. For this purpose we treat two individuals as neighbours in the genotype space if they are separated by a single mutation operation. 
- We consider three different definitions of locality to study which of them gives the best difficulty prediction,

- We consider a mutation based GP (six different mutation operators), without crossover,

- We use three different encodings on five different problems and compare the results against the three definitions of locality, and

- Finally, we use three different distance measures to study genotypic step-size.

This paper is organised as follows. In the following section, a more detailed description on locality will be provided. Section 3 presents previous work on performance prediction. Section 4 presents the experimental setup used to conduct our experiments. In Sect. 5 we present and discuss our findings. Finally, in Sect. 6 we draw some conclusions and in Sect. 7 we outlined future work.

\section{Locality}

Understanding how well neighbouring genotypes correspond to neighbouring phenotypes is a key element in understanding evolutionary search $[52,56]$. In the abstract sense, a mapping has locality if neighbourhood is preserved under that mapping. ${ }^{1}$ In EC this generally refers to the mapping from genotype to phenotype. This is a topic worthy of study because if neighbourhood is not preserved, then the algorithm's attempts to exploit the information provided by an individual's fitness will be misled when the individual's neighbours turn out to be very different.

Rothlauf gives a quantitative definition of locality: "the locality $d_{m}$ of a representation can be defined as

$$
d_{m}=\sum_{d^{g}(x, y)=d_{\min }^{g}}\left|d^{p}(x, y)-d_{\min }^{p}\right|
$$

where $d^{p}(x, y)$ is the phenotypic distance between the phenotypes $x$ and $y, d^{g}(x, y)$ is the genotypic distance between the corresponding genotypes, and $d_{\min }^{p}$ resp. $d_{\min }^{g}$ is the minimum distance between two (neighbouring) phenotypes, resp. genotypes" ([52], p. 77; notation changed slightly). Locality is thus seen as a continuous property rather than a binary one. The point of this definition is that it provides a single quantity which gives an indication of the behaviour of the genotypephenotype mapping and can be compared between different representations.

It should be noticed that while Rothlauf gives a quantitative definition of locality, as expressed above $\left(d_{m}\right)$, this, in fact measures phenotypic divergence. Once a value is obtained by this measure, then we can talk about high and low locality. Although Rothlauf does not provide a threshold value to distinguish high and low locality, nevertheless it is possible to make relative comparisons between representations. As mentioned in Sect. 1, it is important to avoid confusion on the definitions used in this paper. So, we have decided to re-label these two terms. That is, when the phenotypic

\footnotetext{
${ }^{1}$ The term locality has also been used in an unrelated context, to refer to the quasi-geographical distribution of an EC population [8].
} 
divergence $d_{m}$ is low, we are in presence of locality (Routhlauf originally called it high-locality). On the other hand, when the phenotypic divergence $d_{m}$ is high, we are in the presence of "non-locality" (originally called low-locality [52]).

Rothlauf claims that a representation that has locality will be more efficient at evolutionary search. If a representation has locality (i.e., neighbouring genotypes correspond to neighbouring phenotypes) then performance is good.

This, however, changes when a representation has non-locality. To explain how non-locality affects evolution, Rothlauf considered problems in three categories, taken from the work presented in [30]. These are:

- easy, in which fitness increases as the global optimum approaches,

- difficult, for which there is no correlation between fitness and distance and,

- misleading, in which fitness tends to increase with the distance from the global optimum.

It should be noticed that Rothlauf used these three categories only to highlight the implications of the type of locality on these scenarios. To this end, Rothlauf described the following three scenarios with different types of locality.

If a given problem lies in the first category (i.e., easy), a non-locality representation will change this situation by making it more difficult and now, the problem will lie in the second category. This is because non-locality introduces randomness to the search. This can be explained by the fact that representations with non-locality lead to uncorrelated fitness landscapes, so it is difficult for heuristics to extract information.

If a problem lies in the second category, a non-locality representation does not change the difficulty of the problem. There are representations that can convert a problem from difficult (class two) to easy (class one). However, it is non-trivial to construct such a representation.

Finally, if the problem lies in the third category, a representation with nonlocality will transform it so that the problem will lie in the second category. That is, the problem is less difficult because the search has become more random. This is a mirror image of a problem lying in the first category and using a representation that has non-locality.

According to Rothlauf ([52], p. 73) "Previous work has indicated that high locality of a representation is necessary for efficient evolutionary search [23-25, 54, 55] However, it is unclear as to how the locality of a representation influences problem difficulty, and if high locality representations always aid evolutionary search". In this study, as mentioned previously, we want to corroborate this by using locality as a tool for prediction of performance. Before doing so, it is necessary to extend the typical definition of locality from the genotype-phenotype to the genotype-fitness mapping. This extension is presented in the following section.

\subsection{Extending the definition of locality to the genotype-fitness mapping}

Some GP variants have distinct genotypes and phenotypes, for example Cartesian GP [40], linear GP [3], and others. The genotype must be transformed to produce the program proper. The case of standard, tree-structured GP is different, because the genotype is the program proper. Depending on one's viewpoint, one might say that 
the genetic operators work directly on the phenotype, that the genotype-phenotype mapping is the identity map, or simply that no phenotype exists. In this work, we have adopted the traditional GP system, where there is no explicit genotypephenotype mapping, so we can say that there are no explicit phenotypes distinct from genotypes. It is common therefore to study instead the behaviour of the mapping from genotype to fitness [34], and we take this approach here. We will also regard two individuals as neighbours in the genotype space if they are separated by a single mutation. That is, a mutation operator defines the neighbourhood of individuals in the genotype space, adhering to the Jones principle [30] that each operator induces its own landscape.

We begin by considering again the standard definition of locality. It can be characterised as "the sum of surplus distances", where a surplus distance is the actual phenotypic distance between genotypic neighbours less what that distance "should have been". Note in particular Rothlauf's treatment of neutrality. Where genotypic neighbours turn out to lead to the same phenotype, the phenotypic minimum distance $d_{\text {min }}$ is added to the sum $d_{m}$. This is the same quantity that is added when neighbouring genotypes diverge slightly (for bitstring phenotypes with Hamming distance). In other words synonymous redundancy (redundancy of genotypic neighbours) has the same effect as a small divergence of genotypic neighbours. Whether neutrality is beneficial in general is a complex question considered in detail in some previous work [11, 12, 16, 19, 37, 44, 45, 61]; this issue deserves consideration as we will see. The situation is summarised (for discretevalued phenotypic distances) in Table 1 . Thus the final quantity is a continuous measure of the phenotypic divergence and (to a lesser extent) redundancy of genotypic neighbours.

Before explaining how we have extended the definition of locality, first used in GAs, to the genotype-fitness mapping in GP, it is important to state clearly some key elements that we have used in our work.

Firstly, it should be recall that we considered individuals to be genotypic neighbours if a single mutation can transform one into the other. From this we consider whether genotypic neighbours turn out to be fitness neighbours. Given this, it is clear that we need to properly define fitness neighbour.

Recall that locality refers to how well neighbouring genotypes correspond to neighbouring phenotypes and that a representation that shows to preserving good neighbourhood is preferred. This intuitively means that a minimum fitness distance

Table 1 Impact of different phenotypic distances in Rothlauf's definition of genotype-phenotype locality

\begin{tabular}{ll}
\hline Phenotypic distance & Contribution to locality-sum \\
\hline 0 & Small (non-local) \\
Almost 1 & Zero (local) \\
$>>1$ & Non-zero, potentially large (non-local) \\
\hline
\end{tabular}

When genotypic neighbours correspond to phenotypes which differ by the amount shown, the contribution to $d_{m}$ is as shown in column 2 . Recall that in $d_{m}$, smaller is better 
Table 2 Descriptive summary of the contribution to $d_{m}$ made by mutations falling into three classes $(f d=0, f d=1$, and $f d>1)$, for each of three possible definitions of locality

\begin{tabular}{llll}
\hline $\begin{array}{l}\text { Definition } \\
\text { Phenotypic distance }\end{array}$ & $\begin{array}{l}\text { Def }_{0} \\
\text { Contribution }\end{array}$ & Def $_{1}$ & Def $_{2}$ \\
\hline$f d=0$ & Local & Non-local & Local \\
$f d=1$ & Non-local (small) & Local & Local \\
$f d>1$ & Non-local (large) & Non-local & Non-local \\
\hline
\end{tabular}

Discrete case

is required. For instance, for discrete values a minimum fitness distance can be regarded as 0 or 1 . It should be noticed that it is possible to extend this to continues values, as described later in this section. Now, based on the discussion presented in the previous paragraphs regarding how Rothlauf defined locality, the key question is whether to regard genotypic neighbours whose fitness distances are 0 and 1 to be instances of neutrality, locality, or non-locality. The three alternative definitions (summarised in Table 2) are:

- The most straightforward extension of Rothlauf's definition might regard two individuals as fitness-neighbours if the difference of their fitness values is 1 , and regard fitness-neutral mutations as non-local. This leads to the following definition for "non-locality" which we call $\operatorname{Def}_{0}$ (the second column of Table 2).

$$
d_{m}=\frac{\sum_{i=1}^{N}\left|f d\left(x_{i}, m\left(x_{i}\right)\right)-f d_{\min }\right|}{N}
$$

where $f d\left(x_{i}, m\left(x_{i}\right)\right)=\left|f\left(x_{i}\right)-f\left(m\left(x_{i}\right)\right)\right|$ is the fitness distance between a randomly-sampled individual $x_{i}$ and the mutated individual $m\left(x_{i}\right), f d_{\min }=1$ is the minimum fitness distance between two individuals, and $N$ is the sample size.

- However, the above definition treats a fitness-neutral mutation as being just as bad for locality as a mutation causing a fitness divergence of two fitness units (assuming integer-valued fitness). It might be preferable to redefine the minimum distance in the fitness space as zero, giving the same locality definition as above but with $f d_{\min }=0$. This, we term $\operatorname{Def}_{1}$ (the third column of Table 2).

- Finally, it might be better to treat only true divergence of fitness as indicating poor locality. Therefore we might say that fitness divergence occurs only when the fitness distance between the pair of individuals is 2 or greater: otherwise the individuals are regarded as neighbours in the fitness space. This leads to the following definition, which we will call $\operatorname{Def}_{2}$ (the final column of Table 2).

$$
d_{m}=\frac{\sum_{i=1: f d\left(x_{i}, m\left(x_{i}\right)\right) \geq 2}^{N} f d\left(x_{i}, m\left(x_{i}\right)\right)}{N}
$$

Since we have no a priori reason to decide which of these three is the best definition of genotype-fitness locality, we will decide the issue by relating the values produced by each with performance achieved on extensive EC runs. 


\subsection{Generalisation of genotype-fitness locality to continuous-valued fitness}

Several aspects of Rothlauf's definition and the extensions to it given above assume that phenotypic and fitness distances are discrete-valued. In particular, it is assumed that a minimum distance exists. For GP problems of continuous-valued fitness, such as many symbolic regression problems, it is necessary to generalise the above definitions.

- Under the first definition $\left(\operatorname{Def}_{0}\right)$, the quantity being calculated is simply the mean fitness distance. This idea carries over directly to the continuous case.

- Under the second definition $\left(\operatorname{Def}_{1}\right)$, the idea is that mutations should create fitness distances of 1: lesser fitness distances are non-local, as are greater ones. In the continuous case we set up bounds, $\alpha$ and $\beta$, and say that mutations should create fitness distances $\alpha<f d<\beta$. When $f d<\alpha$, we add $\alpha-f d$ to $d_{m}$, penalising an overly-neutral mutation; when $f d>\beta$, we add $f d-\beta$ to $d_{m}$, penalising a highly non-local mutation. Each of these conditions reflects a similar action in the discrete case.

- Under the third definition $\left(\operatorname{Def}_{2}\right)$, both neutral and small non-zero fitness distances are regarded as local; only large fitness distances contribute to $d_{m}$. Thus, in the continuous case, only when $f d>\beta$ do we add a quantity $(f d-\beta)$ to $d_{m}$, penalising mutations of relatively large fitness distances.

Table 3 preserves the same pattern of contributions to $d_{m}$ for continuous-valued fitness as held for discrete-valued fitness, and gives new, generalised definitions based on the idea of two threshold values. $\alpha$ is a small value: mutations which alter fitness by less than $\alpha$ are regarded as essentially fitness-neutral. $\beta$ is larger: mutations which alter fitness by more than $\beta$ are regarded as quite non-local. So, Table 3 generalises Table 2 .

As stated in Sect. 2, Rothlauf's definition of locality is small for well-behaved mappings (locality), and large for badly-behaved mappings (non-locality). This property is inherited by our modifications to the definition.

In the following section, we present some previous work related to problem hardness in EC that inspired our work.

Table 3 Descriptive summary of the contribution to $d_{m}$ made by mutations falling into three classes $(f d<\alpha, \alpha \leq f d<\beta$, and $f d>\beta$ ), for each of three possible definitions of locality

\begin{tabular}{llll}
\hline $\begin{array}{l}\text { Definition } \\
\text { Phenotypic distance }\end{array}$ & $\begin{array}{l}\text { Def } \\
\text { Contribution }\end{array}$ & Def $_{1}$ & Def $_{2}$ \\
\hline$f d<\alpha$ & Non-local (very small); local for $f d=0$ & Non-local & Local \\
$\alpha \leq f d<\beta$ & Non-local (small) & Local & Local \\
$\beta \leq f d$ & Non-local (large) & Non-local & Non-local \\
\hline
\end{tabular}

Continuous case. Note that the pattern of contributions is identical to that of the discrete case 


\section{Related work}

Landscapes and problem difficulty have been the subject of a good deal of research in EC in general and GP in particular. Several approaches to investigating problem difficulty have been proposed. In this section we mention some of them, including their pros and cons.

\subsection{Fitness distance correlation}

Jones [30, 31] proposed the fitness distance correlation (fdc) to measure the difficulty of a problem on the basis of the relationship between fitness and distance to the goal. The idea behind $f d c$ was to consider fitness functions as heuristic functions and to interpret their results as indicators of the distance to the nearest optimum of the search space. $f d c$ is an algebraic measure intended to express the degree to which the fitness function conveys information about distance to the optimum.

According to Jones $[30,31]$ a problem can be classified in one of three classes, depending on the value of $f d c$ :

1. misleading $(f d c \geq 0.15$ ), in which fitness tends to increase with the distance from the global optimum;

2. difficult $(-0.15<f d c<0.15)$, for which there is no correlation between fitness and distance; and

3. easy $(f d c \leq-0.15)$, in which fitness increases as the global optimum approaches.

The threshold interval $[-0.15,0.15]$ was empirically determined by Jones. In $[30,31]$, Jones also proposed the use of scatter plots of distance versus fitness when $f d c$ does not give enough information about the hardness of a problem.

Altenberg [1] argued that predicting the hardness of a problem when using only fitness and distance in an EC system presents some difficulties. For instance, neither crossover nor mutation are taken into account when $f d c$ is calculated, unless their effects are built-in to the measure of genetic distance used. Other works have also shown some weaknesses in $f d c$ [5, 49]. Both [60] and [41] construct examples which demonstrate that the $f d c$ can be "blinded" by particular qualities of the search space, and that it can be misleading. There is, however, a vast amount of work where Jones' approach has been successfully used in a wide variety of problems [12, 16, $44,45]$. Of particular interest is the work by Vanneschi and colleagues [62, 65] which concentrated on the use of $f d c$ in the context of GP. Recent works using $f d c$ on GP include [11, 12]. This is particularly interesting because it has been shown that $f d c$ can be applied using tree-like structures providing a suitable tree distance definition.

\subsection{Fitness clouds and negative slope coefficient}

Later work by Vanneschi and colleagues attempted to address weaknesses of the $f d c$ with new approaches. Fitness clouds plots the relationship between the individuals' 
fitness values in a sample and the fitness of (a sample of) their neighbours. The negative slope coefficient $[64,66]$ can be calculated even without knowing the optima's genotypes and it does not (explicitly) use any distance. In [62, 64], the authors reported good results on some GP benchmark problems. Successively in [63] these results have been extended to some real-like applications. Then in [47] the authors gave a formal model of the fitness proportional negative slope coefficient and a more rigorous justification of it. Finally, in [67], the authors pointed out the limitations of this approach.

\subsection{Other landscape measures}

Several other approaches to studying landscapes and problem difficulty have also been proposed, generally in a non-GP context, including: other measures of landscape correlation [68], autocorrelation [39]; epistasis, which measures the degree of interaction between genes and is a component of deception [20, 21, 41]; monotonicity, which is similar to $f d c$ in that it measures how often fitness improves despite distance to the optimum increasing [41]; and distance distortion which relates overall distance in the genotype and phenotype spaces [52]. All of these measures are to some extent related.

\subsection{Further comments on locality}

Routhlauf [52] was, perhaps, one of the first researchers that formally introduced the concept of locality in Evolutionary Computation systems. However, it is fair to say that other researchers $[10,39]$ have also studied this concept motivated by the same idea: small changes at the genotype level should correspond to small changes at the phenotype level to have locality (originally referred as "high locality" in Routhlauf's work [52]).

Similarly, the principle of strong causality [50] states that for successful search, a small change in genotype should result in a small change in fitness [2, 51]. In other words, the design process of an algorithm should be guided by the locality principle.

\section{Experimental setup}

For our analysis, we have used five well-known problems for GP: the Even- $n$-Parity $(n=\{3,4\})$ problem (problems that require the combination of several XOR functions, and are difficult if no bias favorable to their induction is added in any part of the algorithm), the Artificial Ant Problem [33] (which has been shown to have multimodal deceptive features [36, Chapter 9]) and Real-Valued Symbolic Regression problems (with target functions: $F_{1}=x^{4}+x^{3}+x^{2}+x$ and $\left.F_{2}=2 \sin (x) \cos (y)\right)$.

The first and second problems are Boolean Even- $n$-Parity problems $(n=\{3,4\})$ where the goal is to evolve a function that returns true if an even number of the inputs evaluate to true, and false otherwise. The maximum fitness for this type of problem is $2^{n}$. The terminal set is the set of inputs. In the next section we further 
describe and explain two function sets used in this type of problem to study the locality present.

The third problem, the Artificial Ant Problem [33, pp. 147-155], consists of finding a program that can successfully navigate an artificial ant along a path of 89 pellets of food on a $32 \times 32$ toroidal grid. When the ant encounters a food pellet, its (raw) fitness increases by one, to a maximum of 89 . The problem is in itself challenging for many reasons. The ant must eat all the food pellets (normally in 600 steps) scattered along a twisted track that has single, double and triple gaps along it. The terminal set used for this problem is $T=\{$ Move, Right, Left $\}$. The function sets used in this problem are explained in the following section.

The fourth and fifth problem are real-valued symbolic regression problems. The goal of this type of problem is to find a program whose output is equal to the values of functions. In this case we used functions $F_{1}=x^{4}+x^{3}+x^{2}+x$ and $F_{2}=2 \sin (x) 2 \cos (y)$. Thus, the fitness of an individual in the population reflects how close the output of an individual comes to the target $\left(F_{1}, F_{2}\right)$. It is common to define the fitness as the sum of absolute errors measured at different values of the independent variable $x$, in this case in the range $[-1.0,1.0]$. In this study we have measured the errors for $x \in\{-1.0,-0.9,-0.8 \ldots 0.8,0.9,1.0\}$. We have defined an arbitrary threshold of 0.01 to indicate that an individual with a fitness less than the threshold is regarded as a correct solution, i.e. a "hit". Different threshold values produce different results when comparing the number of hits.

Note that in all five problems, fitness is maximised.

To study locality we need to have alternative representations with differing locality and (presumably) differing performance. Therefore, we will use contrasts in encoding (three encodings: standard GP, alternative function set and a slightly modified encoding) and in operators (six different mutation operators). The idea here is that each encoding will give a different value for locality and different performance, allowing us to compare the predictions of relative performance made by locality with results of evolutionary runs.

\subsection{Alternative function sets}

As mentioned previously, we expect to find different performance when using different function sets. This will allow us to compare the performance predictions made by locality with actual performance. So, for each of the problems used, we propose to use an alternative function set.

Let us start with the Even-n-Parity problem. The terminal set used for this problem is the set of inputs, often called $T=\left\{D_{0}, D_{1}, \ldots, D_{n-1}\right\}$. The standard function set is $F_{E 3}=\{N O T, O R, A N D\}$ and we propose an alternative function set for comparison: $F_{E 4}=\{A N D, O R, N A N D, N O R\}$.

For the Artificial Ant problem, the terminal set used is $T=\{$ Move, Right, Left $\}$. The standard function set is $F_{A 3}=\{I f, P 2, P 3\}$ (see [33] for a full description). In order to have alternative encodings (with again, as we will see, different performance and locality characteristics), we now propose an alternative function set. This is $F_{A 4}=\{I f, P 2, P 3, P 4\}$. The only difference is the addition of an extra sequencing function, $\mathrm{P} 4$, which runs each of its four subtree arguments in order. 
For the last type of problem, Symbolic Regression, the terminal set is defined by the variables used in the function (e.g., $T=\{x\}$ ). The standard function set $F_{S 6}=\{+,-, *, \%, \operatorname{Sin}, \operatorname{Cos}\}$, and we propose an alternative function set for comparison purposes: $F_{S 4}=\{+,-, *, \%\}$.

\subsection{Uniform GP}

In our previous work $[13,14]$, we have studied contrasts between GP encodings induced by using alternative function sets. However, in GP contrasts also exist between different encodings, including linear GP [42], graph-based GP [18], and more. In order to include in our study the distinction between encodings, we use a contrast between standard tree-structured GP and a slightly different GP encoding called Uniform GP [17].

Uniform GP, called uniform because all internal nodes are of the same arity, is an encoding defined by adding 'dummy' arguments (i.e., terminals/subtrees) to internal nodes whose arity is lower than the maximum arity defined in the function set. These are called dummy arguments because they are not executed when the individual is evaluated. For this to happen, it is necessary to use special functions that indicate the use of dummy arguments (these special functions can be seen as flags that indicate that the subtree beneath these type of functions will not be executed). Thus, dummy arguments can be seen only in trees that allow those special functions.

For a better understanding of how this representation works, let us present an example. Suppose that one is dealing with the traditional Artificial Ant Problem. That is, the function set is formed by $F=\{I f, P 2, P 3\}$ where the arities of each function are 2, 2 and 3, respectively. This function set has a maximum arity of 3 . A typical GP individual is shown in Fig. 1 (left) and a corresponding individual using Uniform GP is shown on the right of Fig. $1 .^{2}$ Please, refer to [17] for a detailed explanation.

To use this representation in our five problems, we defined three function sets: $F_{E 3^{*}}=\{A N D, O R, N O T 2\}, F_{A 3^{*}}=\{$ If $3, P 23, P 3\} \quad$ and $\quad F_{S 6^{*}}=\{+,-, *, \%, \operatorname{Sin} 2$, $\operatorname{Cos} 2\}$ (where $\%$ is protected division), for the Even- $n$-Parity $(n=\{3,4\})$, Artificial Ant, and Symbolic Regression problems $\left(F_{1}=x^{4}+x^{3}+x^{2}+x\right.$, $\left.F_{2}=2 \sin (x) \cos (y)\right)$, respectively. NOT2, P23, $\operatorname{Sin} 2, \operatorname{Cos} 2$ allow the addition of dummy arguments as explained previously (an example can be seen in Fig. 1).

Table 4 shows all the function sets declared for each of the problems used in this study. Note that those marked with $*$ indicate the use of Uniform GP.

\subsection{Mutation operators}

As mentioned in Sect. 1, we regard two individuals as neighbours in the genotype space if they are separated by a single mutation. We used six different mutation operators in our studies, taken from the specialised literature [46] where one-point

\footnotetext{
${ }^{2}$ Notice that this type of encoding is distinct only when the arities defined in the function set are of different values. So, if arities are all the same, Uniform GP reduces to standard GP.
} 

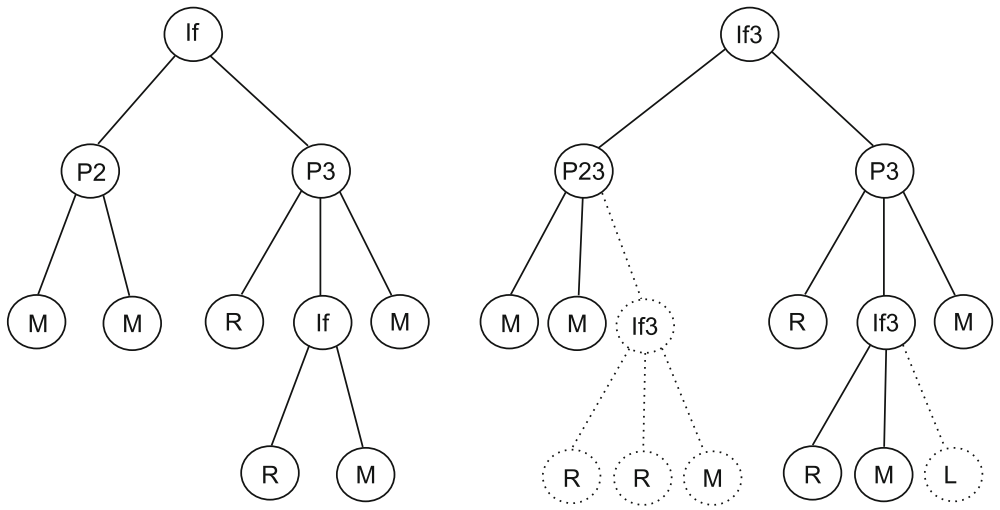

Fig. 1 A typical GP individual (left) and the same individual with uniform arity 3 (right). Dashed lines indicate dummy arguments

Table 4 Function sets used on the Even- $n$-Parity Problem $(n=\{3,4\})$, the Artificial Ant problem and symbolic regression problems $\left(F_{1}=x^{4},+x^{3}+x^{2}+x, F_{2}=2 \sin (x) \cos (y)\right)$

\begin{tabular}{ll}
\hline Problem & Function sets \\
\hline Even- $n$-parity & $F_{E 3}=\{A N D$, OR,NOT $\}$ \\
& $F_{E 4}=\{A N D$, OR,NAND, NOR $\}$ \\
& $F_{E 3^{*}}=\{A N D, O R, N O T 2\}$ \\
Artificial Ant & $F_{A 3}=\{I F, P 2, P 3\}$ \\
& $F_{A 4}=\{I F, P 2, P 3, P 4\}$ \\
Symbolic regression & $F_{A 3^{*}}=\{I F 3, P 23, P 3\}$ \\
& $F_{S 6}=\{+,-, *, \%, \operatorname{Sin}, \operatorname{Cos}\}$ \\
& $F_{S 4}=\{+,-, *, \%\}$ \\
& $F_{S 6^{*}}=\{+,-, *, \%, \operatorname{Sin} 2, \operatorname{Cos} 2\}$ \\
&
\end{tabular}

$F_{E 3^{*}}, F_{A 3^{*}}$ and $F_{S 6^{*}}$ refer to the Uniform GP (read text)

and subtree mutation are the most popular mutations operators used within the GP community. ${ }^{3}$ These are:

1. One-Point mutation, also known as node replacement mutation, replaces a node (leaf or internal) in the individual by a new node chosen randomly among those of the same arity to ensure that the expression remains valid, taking the arity of a leaf as zero.

2. Subtree mutation replaces a randomly selected subtree with another randomly created subtree as proposed in ([33], p. 106).

3. Permutation mutation creates a new individual by selecting randomly an internal node and then randomly permuting its arguments [33].

4. Hoist mutation creates a new individual. The resulting offspring is a copy of a randomly chosen subtree of the parent [32].

\footnotetext{
3 Notice that Size-fair subtree mutation has two variants.
} 
5. Size-fair subtree mutation was proposed by Langdon [35] with two variants. For the first method, the size of the new individual is given by the size $s$ of a subtree chosen at random within the parent. Size $s$ is then used for creating a new individual randomly. For the second method, the size of the replacement subree is chosen uniformly in the range $[l / 2,3 l / 2]$ (where $l$ is the size of the subtree being replaced). In his experiments, Langdon showed that the former method produced far more bloat compared to the second method.

\subsection{Sampling and parameters}

To have sufficient statistical data, we created 1,250,000 individuals for each of the six mutation operators described previously. These samplings were created using the traditional ramped half-and-half initialisation method described in [33] using depths $=[3,8]$. By using this method, we guarantee that we will use trees of different sizes and shapes.

\subsubsection{Comments on the sampling method}

This sampling method is only one possibility among many. Another approach that can be considered is to balance the number of bad individuals (low fitness values) against good individuals (fitter individuals) by creating a sample for each of the individuals that will be inserted in the final sampling. That is, one could take the approach of sampling individuals, take the fitter value(s) and add those into the final sampling, and repeating this process until the final sampling has been completed. Another approach that one can use is generating the same number of individuals for each of the fitness values that can be created, as shown in our previous work [15]. There are some restrictions with this approach. For instance, one should know in advance all the possible values that the fitness could take. Also, the generation of individuals with high fitness is very hard to achieve. Other approaches might be possible and each of these will definitely give different results.

We decided to start with one the simplest possible approaches to generate a sampling. In future work, we would like to explore the implications of using different sampling methods.

\subsection{Studying locality}

To study and examine the locality present, for each data point in the sample data, we created an offspring via each mutation operator described earlier, as in our locality definitions (Sect. 2).

To compare the predictions made by locality, we performed runs using the parameters shown in Table 5 and using the function sets explained previously and summarised in Table 4. In particular, note that mutation-only GP was used. This is because in typical EC scenarios including crossover, mutation is relegated to a "background operator" [27]. In such an algorithm, the true neighbourhood of individuals is largely determined by their mutual accessibility under crossover, 
Table 5 Parameters used to conduct our experiments

\begin{tabular}{ll}
\hline Selection & Tournament (size 7) \\
Initial population & Ramped half and half (depth 3 to 8) \\
Population size & $200,250,500$ \\
Generations & $125,100,50(25,000$ divided by \\
& population size) \\
Runs & 100 \\
Mutations & One point, subtree, permutation, \\
& hoist size-fair \& size-fair range \\
Mutation rate & One single mutation per individual \\
Termination & Maximum number of generations \\
\hline
\end{tabular}

Notice that we used different combinations of population sizes along with number of generations

rather than mutation, and so the typical, mutation-based definition of locality used here would be misleading.

In the following section we present and describe the results on locality using the described parameters.

\section{Results}

\subsection{Fitness distance distributions}

Let us start our analysis by taking a look at the distributions of fitness distances $(f d)$ induced by the six mutation operators explained in Sect. 4. In particular, we will focus our attention on $f d=\{0,1\}$ for discrete values (i.e., Even- $n$-Parity and Artificial Ant). This situation, however, changes for continuous values which is the case for the Symbolic Regression problem. Here, as explained in Sect. 2, it is necessary to set thresholds (denoted with $\alpha$ and $\beta$ ). So, we analyse the frequency of fitness distance grouped into bins $(f d<\alpha, \alpha<f d<\beta)$. Figure 2 shows the frequency of each possible $f d$ between parent-offspring, for the five problems used in this study (introduced in Sect. 4) and three function sets (see Table 4) for each problem.

For the first and second problem, Even- $n$-Parity $(n=\{3,4\})$ problems, we can see on the fitness distance distribution (first and second row of Fig. 2) that regardless of the encoding used a high number of mutations are fitness neutral (i.e., $f d=0$ ). There are, however, some variations worth mentioning. For instance, $F_{E 3}$ and $F_{E 3^{*}}$ (see Table 4 for a description of them) produce the largest number of neutral mutations for all six mutation operators used ${ }^{4}$ compared with $F_{E 4}$. If we further analyse this fitness neutrality in each of the function sets, we can see that one-point mutation produces the largest number of neutral $(f d=0)$ mutations compared to the other mutation operators (excluding permutation mutation for this particular problem for the reasons explained previously). On the other hand, we can see that $F_{E 4}$ produces the largest number of $(f d=1)$ mutations compared to $F_{E 3}$ and $F_{E 3^{*}}$.

\footnotetext{
${ }^{4}$ Notice that when using the permutation mutation operator and using $F_{E 3}$ and $F_{E 4}$ on the Even- $n$-Parity problem, the $f d$ is always 0 because all of the operators in these function sets are symmetric.
} 

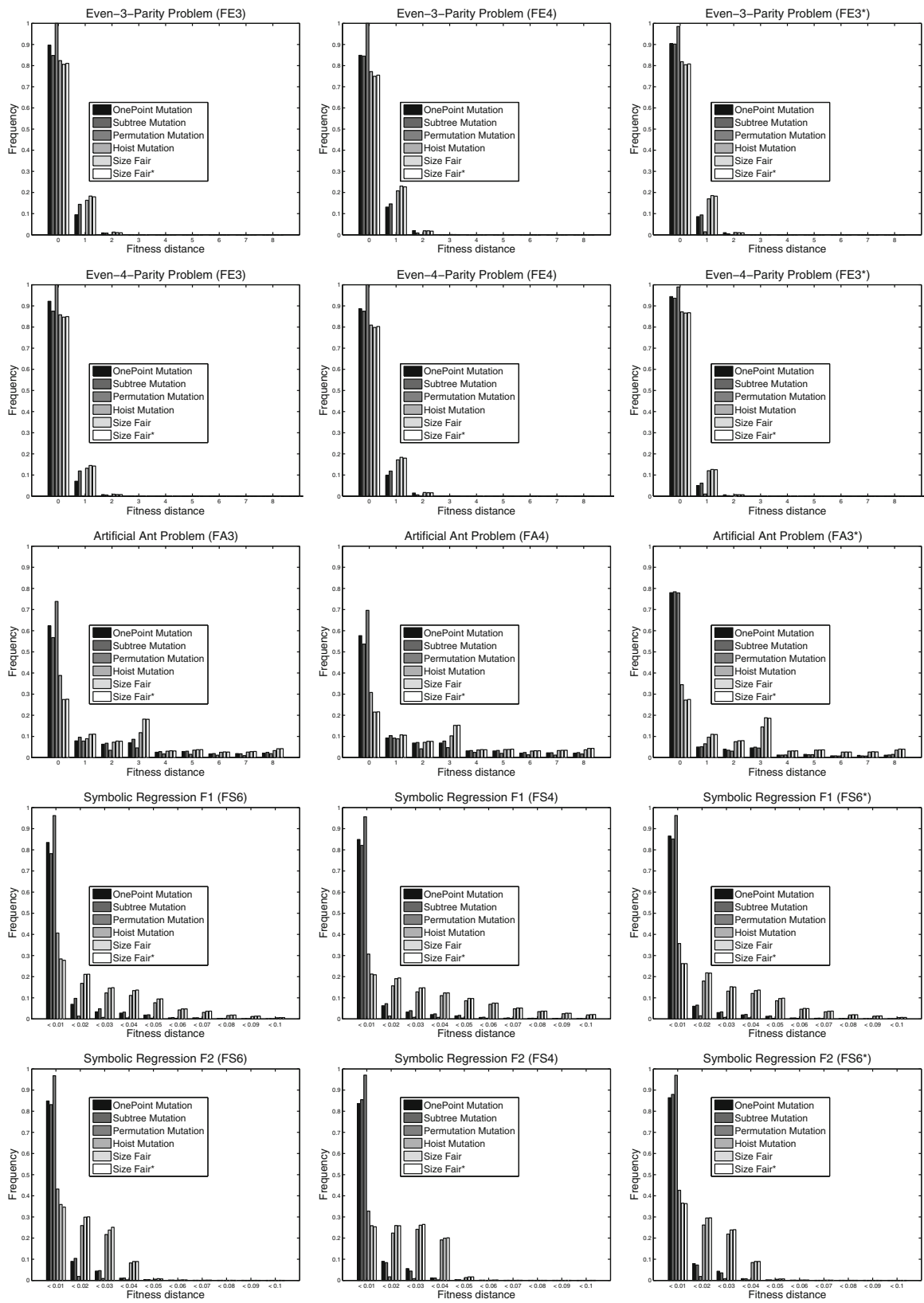

Fig. 2 Distribution of fitness distance values on the Even-3, Even-4, the Artificial Ant, and two symbolic regression problems $\left(F_{1}\right.$ and $\left.F_{2}\right)$ from top to bottom using six mutation operators, with standard function set (left), alternative function set (centre) and a function set for Uniform GP (right) 
For the third problem, the Artificial Ant, fitness differences of up to 89 are possible, but larger values are rare and their frequency decreases roughly linearly. Therefore, we have omitted values above 8 to make the important values easier to visualise (see middle of Fig. 2). Again, we can see that regardless of the encoding used, a high number of mutations are fitness neutral, where the largest number of occurrences of $f d=0$ is when using the encoding $F_{A 3 *}$ and subtree mutation. For the case of $f d=1$, the situation is less clear. Here, using any of the three encodings (i.e., $\left.F_{A 3}, F_{A 4}, F_{A 3 *}\right)$ seems to produce more or less the same number of occurrences.

For the last two problems, Symbolic Regression $\left(F_{1}\right.$ and $\left.F_{2}\right)$ functions, it is no longer possible to use the same approach as before because of the nature of the problem (continuous-valued fitness). So, as mentioned previously, we analyse the frequency of fitness distances grouped into bins. For this particular problem and for the threshold values that we have used for our studies, it is not clear what encoding (induced by $F_{S 6}, F_{S 4}$ and $F_{S 6^{*}}$ ) produces the largest number of occurrences for $f d<\alpha$ and $\alpha<f d<\beta$. In the following section we further discuss this and clarify the type of locality present in this problem.

Finally, it should be noticed that Uniform GP (introduced in Sect. 4) was originally proposed to add neutrality in the search space, as explained in [17]. So, we can see that in almost all cases, this type of encoding produced a larger number of neutral mutations compared to the other two alternative function sets (see third column of Fig. 2).

It is clear that while fitness distance distributions can give us an idea of the type of locality induced by each mutation operator and encoding, the plots shown in Fig. 2 cannot really tell us which operator and encoding gives the best locality. Thus, to better understand this, one should really take a look at the quantitative measures introduced formally in Sect. 2. This will be discussed in the next section.

\subsection{Quantitative measures and performance}

As mentioned previously (see Sect. 2), Rothlauf [52] distinguished two forms of locality: high and low locality. In a nutshell, Rothlauf claimed that a representation with high locality is more likely to perform effective evolutionary search compared to a representation with low locality. However, his numerical definition, and the extensions we have proposed for it, give smaller values for higher locality. To avoid confusion, as we mentioned previously, we refer to high and low locality as locality and non-locality, respectively.

We have seen in the previous paragraphs an overall picture of the fitness distance distributions induced by different encodings on the five problems analysed in this paper. As mentioned previously, to better understand the effects of locality on performance, a quantitative analysis is necessary. To achieve this, we performed extensive empirical experimentation $(100 \times 45 \times 6 \text { runs in total })^{5}$. Details of the parameters used to conduct our runs are shown in Table 5. Because of the

\footnotetext{
5100 independent runs, 45 different settings (i.e., three different combinations of population sizes and number of generations, five different problems and three different function sets for each of the five problems $-3 \times 5 \times 3$ ), and 6 different mutation operators.
} 
Table 6 Number of correct predictions of good locality on performance (measured as mean best fitness over 100 runs), for the Even- $n$-Parity $(n=\{3,4\})$, Artificial Ant and two Symbolic Regression problems $\left(F_{1}, F_{2}\right)$ and using six different mutation operators

\begin{tabular}{|c|c|c|c|c|c|c|c|}
\hline & One Point & Subtree & Permut. & Hoist & Size Fair & Size Fair* & Total \\
\hline & \multicolumn{7}{|c|}{ Even-3-Parity } \\
\hline $\operatorname{Def}_{0}$ & 0 & 0 & - & 2 & 2 & 2 & 6 \\
\hline $\operatorname{Def}_{1}$ & 3 & 3 & - & 1 & 1 & 1 & 9 \\
\hline \multirow[t]{2}{*}{$\mathrm{Def}_{2}$} & 0 & 0 & - & 0 & 0 & 0 & 0 \\
\hline & \multicolumn{7}{|c|}{ Even-4-Parity } \\
\hline $\operatorname{Def}_{0}$ & 1 & 1 & - & 1 & 0 & 1 & 4 \\
\hline $\operatorname{Def}_{1}$ & 1 & 1 & - & 3 & 3 & 3 & 11 \\
\hline \multirow[t]{2}{*}{$\operatorname{Def}_{2}$} & 0 & 1 & - & 0 & 0 & 0 & 1 \\
\hline & \multicolumn{7}{|c|}{ Artificial Ant } \\
\hline $\operatorname{Def}_{0}$ & 0 & 0 & 3 & 0 & 3 & 3 & 9 \\
\hline $\operatorname{Def}_{1}$ & 0 & 0 & 3 & 0 & 3 & 3 & 9 \\
\hline \multirow[t]{2}{*}{$\operatorname{Def}_{2}$} & 0 & 0 & 3 & 0 & 3 & 3 & 9 \\
\hline & \multicolumn{7}{|c|}{ Symbolic regression $F_{1}$} \\
\hline $\operatorname{Def}_{0}$ & 2 & 2 & 0 & 0 & 0 & 0 & 4 \\
\hline $\operatorname{Def}_{1}$ & 0 & 0 & 0 & 3 & 3 & 3 & 9 \\
\hline \multirow[t]{2}{*}{$\mathrm{Def}_{2}$} & 2 & 2 & 0 & 0 & 0 & 0 & 4 \\
\hline & \multicolumn{7}{|c|}{ Symbolic regression $F_{2}$} \\
\hline $\operatorname{Def}_{0}$ & 1 & 1 & 2 & 1 & 1 & 0 & 6 \\
\hline Def $_{1}$ & 3 & 1 & 2 & 1 & 1 & 1 & 9 \\
\hline $\mathrm{Def}_{2}$ & 1 & 1 & 1 & 1 & 1 & 1 & 6 \\
\hline
\end{tabular}

The three different definitions of locality are denoted by $\operatorname{Def}_{0}, \operatorname{Def}_{1}$ and $\operatorname{Def}_{2}$. Table 2 shows the locality definitions for discrete-valued fitness (e.g., Even- $n$-Parity and Artificial Ant). Table 3 shows the locality definitions for continuous-values fitness (e.g., Symbolic regression)

dimensions of all the information gathered during our experiments (details of these results can be seen in "Appendix" from Table 9, 10, 11, 12, 13, 14, 15, 16, 17 and 18), we have decided to process this information to help the reader to better understand our findings.

Let us start with the number of correct predictions of locality on performance on the five problems and six different mutation operators used in this study. This is shown in Table 6. Using the data shown from Tables 9, 10, 11, 12, 13, 14, 15, 16, 17 and 18, this table was built by comparing the best locality (recall that the lower the value for locality, the better) versus performance (measured in terms of best average fitness per run) over three different settings (i.e., different population sizes and number of generations). Thus, a perfect prediction will be 3 , where the representation with the best locality value under a particular definition turns out to give the best performance over all three population size/number of generations settings. We have grouped the three definitions (denoted by $\operatorname{Def}_{0}, \operatorname{Def}_{1}$ and $\operatorname{Def}_{2}$ - see caption of Table 6 for a description of them) for each of the five problems used in this study.

So, let us focus our attention on the Even-3-Parity problem. The results shown in Table 6 indicate that $\operatorname{Def}_{0}$ was able to correctly predict 2 (out of 3 ) when using 
Hoist, Size Fair mutation and its variant. $D e f_{1}$ was able to correctly predict performance when using One-Point and Subtree mutation on the three different settings (i.e., population sizes and number of generations) and was able to predict performance only once when using Hoist, Size Fair mutation and its variant. On the other hand, Def 2 was unable to predict performance of any of the mutation operators used for the Even-3-Parity problem. When we focus our attention on the overall prediction versus performance for each of the definitions of locality (shown at the bottom of Table 6), we can see that the definition of locality that most frequently predicted performance correctly on the Even-3-Parity problem is Def ${ }_{1}$ with 9 correct predictions. It is important to mention that the lack of values for the Permutation operator for this particular problem is due to the nature of both the operator and the problem in itself. That is, recalling how the permutation operator works (described in detail in Sect. 4), we can see that this operator will not have any impact after being applied to an individual because the binary operators AND and OR are insensitive to the ordering of their arguments.

The same trend can be observed for the second problem, the Even-4-Parity. That is, Def $_{1}$ was able to predict correctly 11 out of 15 (recall that a perfect prediction is 3 for each mutation operator - in this particular case only 5 mutation operators because permutation cannot be applied for this problem as explained in the previous paragraph). For Def 0 and Def 2 the overall prediction was 4 and 1, respectively. This agrees with the results on the Even-3-Parity problem, as described previously.

Now, let us turn our attention to the third problem, the Artificial Ant problem. Here, the three definitions of locality (formally introduced and described in Sect. 2) show the same behaviour, so none of the definitions is better or worse than the others.

For the fourth problem Symbolic Regression problem $F_{1}$, definitions $\operatorname{Def}_{0}$ and Def $_{2}$ correctly predicted the performance in 2 out of 3 cases for One-Point and Subtree mutation. On the other hand, Def ${ }_{1}$ had a perfect prediction on performance for Hoist, Size Fair mutation and its variant. Again, when we focus our attention on the overall prediction versus performance for each of the definitions on locality, we can clearly see that the definition of locality that most frequently correctly predicted performance is $\alpha<f d_{\text {min }}<\beta$ (denoted by Def ${ }_{1}$ ) with 9 correct predictions.

Finally, for the last problem (Symbolic Regression problem $F_{2}$ ), we continue seeing the same trend. That is, Def $_{1}$ gives the best prediction with 9, compared to the predictions done by $\operatorname{Def}_{0}$ and $\operatorname{Def}_{2}$ with 6 for these two definitions.

So far, we have examined how many of the "best" locality made a good prediction. In other words, locality should correspond with the best performance (measured in terms of the average of the best fitness per generation). As mentioned previously, we have condensed this information in Table 6 (details can be found from Table 9 to 18 shown in "Appendix") and, as discussed in the previous paragraphs, we can clearly see that overall, the best definition of locality in terms of

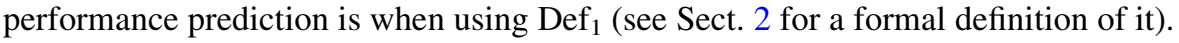

Of course, there are other ways to interpret all the data gathered during our experiments (shown in Tables 9, 10, 11, 12, 13, 14, 15, 16, 17 and 18). So, to corroborate these initial findings, we have analysed all locality values (shown in Tables 9, 11, 13, 15 and 17) and compared their values against performance (shown in Tables 10, 12, 14, 16 and 18) over all the six mutation operators used in this study 
Table 7 Number of correct predictions of all locality values on performance (measured as mean best fitness over 100 runs), for the Even- $n$-Parity $(n=\{3,4\})$, Artificial Ant and two Symbolic Regression problems $\left(F_{1}, F_{2}\right)$

\begin{tabular}{|c|c|c|c|c|}
\hline & $F_{E 3}, F_{A 3}, F_{S 6}$ & $F_{E 4}, F_{A 4}, F_{S 4}$ & $F_{E 3^{*}}, F_{A 3^{*}}, F_{S 6^{*}}$ & Total \\
\hline & \multicolumn{4}{|l|}{ Even-3-Parity } \\
\hline $\operatorname{Def}_{0}$ & 7 & 0 & 2 & 9 \\
\hline $\operatorname{Def}_{1}$ & 3 & 9 & 3 & 15 \\
\hline \multirow[t]{2}{*}{$\mathrm{Def}_{2}$} & 3 & 0 & 3 & 6 \\
\hline & \multicolumn{4}{|l|}{ Even-4-Parity } \\
\hline $\operatorname{Def}_{0}$ & 8 & 2 & 0 & 10 \\
\hline $\operatorname{Def}_{1}$ & 5 & 7 & 11 & 23 \\
\hline \multirow[t]{2}{*}{$\mathrm{Def}_{2}$} & 6 & 2 & 1 & 9 \\
\hline & \multicolumn{4}{|l|}{ Artificial Ant } \\
\hline $\operatorname{Def}_{0}$ & 7 & 12 & 9 & 28 \\
\hline $\operatorname{Def}_{1}$ & 7 & 12 & 9 & 28 \\
\hline \multirow[t]{2}{*}{$\operatorname{Def}_{2}$} & 7 & 12 & 9 & 28 \\
\hline & \multicolumn{4}{|c|}{ Symbolic Regression $F_{1}$} \\
\hline $\operatorname{Def}_{0}$ & 6 & 4 & 12 & 22 \\
\hline $\operatorname{Def}_{1}$ & 10 & 16 & 12 & 38 \\
\hline \multirow[t]{2}{*}{$\mathrm{Def}_{2}$} & 5 & 0 & 4 & 9 \\
\hline & \multicolumn{4}{|c|}{ Symbolic Regression $F_{2}$} \\
\hline $\operatorname{Def}_{0}$ & 3 & 3 & 7 & 13 \\
\hline $\operatorname{Def}_{1}$ & 10 & 2 & 4 & 16 \\
\hline $\mathrm{Def}_{2}$ & 4 & 2 & 5 & 11 \\
\hline
\end{tabular}

The three different definitions of locality are denoted by $\operatorname{Def}_{0}, \operatorname{Def}_{1}$ and Def 2 . Table 2 shows the locality definitions for discrete-valued fitness (e.g., Even- $n$-Parity and Artificial Ant). Table 3 shows the locality definitions for continuous-values fitness (e.g., Symbolic Regression). $F_{E 3}, F_{A 3}, F_{S 6}$ uses standard GP, $F_{E 4}, F_{A 4}, F_{S 4}$ uses an alternative function set and $F_{E 3^{*}}, F_{A 3^{*}}, F_{S 6^{*}}$ uses Uniform GP for the Even- $n$-Parity, Artificial Ant and Symbolic Regression problems, respectively

(see Sect. 4 for a description of each of them). We have condensed this information in a way to highlight what definition of locality is the best focusing on function sets used for each problem and the three definitions of locality used in this study. This data is shown in Table 7.

As mentioned before, we have obtained this data (Table 7) by comparing each value of locality versus performance. To better understand this, let us focus our attention on the Even-3-Parity problem (predictions made by locality are shown in Table 9 and performance is shown in Table 10).

We have obtained this data by comparing each prediction made by locality versus performance, focusing our attention on each of the definition of locality and each of the function sets used. For instance, let us focus our attention on the predictions done by $\mathrm{Def}_{0}$ on the Even-3-Parity (shown in Table 9), using One-Point mutation and $F_{E 3}$. We can see that the quantitative measure is 0.1125 and the other two values for $F_{E 4}$ and $F_{E 3 *}$ are 0.1727 and 0.1059 , respectively. This means, that the best locality is when using $F_{E 3 *}$ (recall that the lower the value of locality, the better). 
The locality value for $F_{E 3}$ is the second best, which means that performance when using One-Point mutation and $F_{E 3}$ should be the second best performance, regardless of the population size and number of generations used. When we check this, we can observe that in none of the cases, this was true. We continue doing the same for the rest of the mutation operators. At the end, we sum all the values (it is clear that we can have up to 18 correct predictions). Finally, to summarise these values we count the number of correct predictions for all the function sets (per row for each of the problems used). These values are shown in the last row of Table 7.

This data (Table 7) simply confirms our previous findings, the best definition of locality (three definition formally introduced in Sect. 2) on the five problems examined in this paper is $\operatorname{Def}_{1}$.

\subsubsection{Correlating quantitative measures and performance}

We can perform another form of analysis on the same data (Tables 9, 10, 11, 12, 13, $14,15,16,17$ and 18), again relating the results of evolutionary runs with those of locality calculations. Here, we calculate the correlation between the mean best fitness values achieved on the various problems, using the various encodings and operators, and the corresponding locality values. If locality values are functioning as predictors of performance, then a correlation between the two should exist. For each problem and each locality-definition, then, we arrange all locality values by function set and mutation. We do the same for performance values, with all population size/ number of generations settings grouped together. We then calculate the Pearson's correlation between these two data-sets.

The results are shown in Table 8 . For the Even- $n$-Parity problems, all three definitions of locality are shown to give values uncorrelated with fitness values (correlations are not statistically significant, $p>0.05$. Therefore, none of our definitions of locality makes useful predictions on these problems.

In the case of the Artificial Ant, all three definitions of locality are shown to give values which are strongly negatively correlated $(p<0.0001)$ with performance. That is, lower (better) values of locality are associated with higher (better) performance. Thus, all three definitions are making good predictions. This matches with our initial findings shown in Tables 6 and 7. Also, this is evidence in favour of locality as a measure of problem difficulty, though no new evidence is found in favour of one definition over the others on this problem.

Finally, in the case of the Symbolic Regression problems, lower values of fitness are better, so a positive correlation indicates good predictions. Def ${ }_{1}$ gives a good result (high positive correlation, $p<0.001$ ); Def $_{0}$ gives a misleading result (a negative

Table 8 Correlation values

\begin{tabular}{|c|c|c|c|c|c|}
\hline Problem def. & Even-3 & Even-4 & Artificial Ant & $F_{1}$ & $F_{2}$ \\
\hline $\operatorname{Def}_{0}$ & $-0.16(\times)$ & $0.11(\times)$ & $-0.73(\boldsymbol{})$ & $-0.34(\times)$ & $-0.36(\times)$ \\
\hline Def $_{1}$ & $0.19(\times)$ & $0.13(\times)$ & $-0.74(レ)$ & $0.47(\swarrow)$ & $0.34(\mathscr{})$ \\
\hline $\mathrm{Def}_{2}$ & $0.01(\times)$ & $-0.02(\times)$ & -0.74 (ん) & $0.05(\times)$ & $0.11(\times)$ \\
\hline
\end{tabular}


correlation), and $\mathrm{Def}_{2}$ 's correlation does not give us information. Thus, we find some evidence in favour of Def $_{1}$. This agrees with the results reported in Tables 6 and 7.

\subsection{Genotypic and fitness distance distributions}

In previous sections we have seen that each mutation operator leads to a characteristic distribution of fitness distances for each problem and each encoding: indeed, it is these distributions which are intended to be summarised by our locality measures. We now give a partial explanation of how the differences between these distributions arise. Recall that our definition of genetic neighbourhood is based on mutation operators: two individuals are neighbours if one can be transformed into the other by a single mutation. Simply, the fitness distance between neighbours arises partly from the effect of the operator (how different are the individuals in genetic space?) and partly from the effect of the genotype-fitness mapping (to what extent does the mapping allow similar individuals to diverge?). Studying the first of these two effects is the goal of this section.

Previous work has studied "the amount of variation generated in the genotype" by an operator application, and the effect of this variation on phenotypic variation [28]. This concept is here termed the genotypic step-size. It can be quantified by applying a distance function to the original and mutated individual [28].

In many EC applications, it is useful to choose a distance function which is coherent with the operator, i.e. that reflects the "remoteness" of individuals via applications of an operator. Distances have been described which reflect the number of operator applications required to produce one individual from another [6, 43, 62, 65] or the probability of producing one individual from another in a single step [26].

Such an approach turns out not to be useful for the current experiment. If a genotypic distance coherent with the operator is used to measure genotypic stepsize, then by definition every pair of neighbours (which differ by a single operator application) will turn out to be at a minimum distance from each other. If we try to compare the genotypic step-sizes of two different operators, for each using a distance coherent with the operator, we will discover that every operator gives pairs of neighbours which are always at the minimum distance from the original, and so we will get a "null" result: every operator achieves the same genotypic step-size.

Instead, it is necessary to distinguish between the ideas of "remoteness" and "dissimilarity". Dissimilarity is a general-purpose concept, unrelated to the process of transforming individuals using operators. In the next section we therefore introduce three general-purpose ways to quantify dissimilarity of GP trees. No claim is made that these three are the "best" ways to quantify dissimilarity. However, by using three different and general-purpose functions, and showing that they do not contradict each other, arguments concerning the size of the change caused by different mutation operators can be convincingly supported.

\subsubsection{Tree distance measures for GP}

There are at least three natural approaches to comparing tree structures, based respectively on minimal editing operations (tree-edit distance), alignment of similar 
structures (tree-alignment distance) and normalised compression distance. Each of these has been studied extensively outside the field of EC [4, 29, 57, 59], and used by EC researchers also $[6,7,9,22,43,60,65]$. We describe them in more detail next.

5.3.1.1 Tree-edit distance Tree-edit distance is integer-valued and reflective of a very intuitive notion of the distance between a pair of trees, based on the number of edits required to transform one into the other. Three types of edits are allowed: insertion, deletion, and substitution. This distance measure was studied outside EC, with algorithms given by [57, 59]. It was proposed for use in GP by [43], and is notable partly because it is closely aligned with a mutation operator defined in the same paper.

5.3.1.2 Tree-alignment distance Tree-alignment distance is a good generalpurpose, continuous-valued measure which reflects the fact that the roots of syntactic trees tend to be more important than their lower levels. This measure, proposed for use in GP by Vanneschi and colleagues [6, 60, 62, 65], is based on that proposed by [29] via [9].

Formally, the distance between trees $T_{1}$ and $T_{2}$ with roots $R_{1}$ and $R_{2}$, respectively, is defined as follows:

$$
\operatorname{dist}\left(T_{1}, T_{2}, k\right)=d\left(R_{1}, R_{2}\right)+k \sum_{i=1}^{m} \operatorname{dist}\left(\operatorname{child}_{i}\left(R_{1}\right), \operatorname{child}_{i}\left(R_{2}\right), \frac{k}{2}\right)
$$

where $d\left(R_{1}, R_{2}\right)=\left(\left|c\left(R_{1}\right)-c\left(R_{2}\right)\right|\right)^{z}$ and $\operatorname{child}_{i}(Y)$ is the ith of the $m$ possible children of a node $Y$, if $i<m$, or the empty tree otherwise. Note that $c$ evaluated on the root of an empty tree is 0 by convention. The parameter $k$ is used to give different weights to nodes belonging to different levels in the tree and $z \in \mathbb{N}$ is a parameter of the distance. The depth-weighting is well-motivated, in that GP trees' roots tend to be more important than their lowest levels. Code for this calculation is available in Galván's Ph.D. thesis [11]. This distance is notable partly because, for $k=1$ (i.e. without depth-weighting) and for a particular function/terminal set (the Royal Tree set [48]), the distance is coherent with a specially-constructed pair of mutation operators. The configuration we use in this paper does use depthweighting, and we are not using the Royal Tree function/terminal set, and so our tree-alignment distance is not coherent with any operator. As demonstrated by Vanneschi and colleagues, it is still useful as a general-purpose distance function.

5.3.1.3 Normalised compression distance The so-called "universal similarity metric" is a theoretical measure of similarity between any two data structures (for example strings), defined in terms of Kolmogorov complexity [38]. This is defined as the length of the shortest program which creates the given string. Informally, two strings are very similar if the Kolmogorov complexity of their concatenation is close to the complexity of just one of them. This idea was made practical by [4]: they approximated the (uncomputable) Kolmogorov complexity of a string by the length of its compressed version, as calculated by off-the-shelf compression software. The "normalised compression distance" or NCD is defined as follows: 


$$
d(x, y)=\frac{C(x y)-\min (C(x), C(y))}{\max (C(x), C(y))}
$$

where $x$ and $y$ are two strings, $x y$ is their concatenation, and the function $C$ gives the length of the compressed version of its argument.

The NCD has been used as a distance measure for trees in fields other than EC [4] and has been used for linear structures within EC [22], and GP has been used to approximate Kolmogorov complexity [7]. However, to the authors' knowledge the NCD has not yet been used to measure distance between GP trees. It can be applied to trees simply by encoding them as strings in prefix format. For fixed node arities, this encoding is injective, i.e. distinct trees will give distinct prefix strings. It has the advantage that a single mutation in a large tree will lead to a smaller distance than a single mutation in a small tree. On the other hand, mutations near the root have the same weight as deeper ones. It shares this disadvantage with, for example, tree edit distance. Such distances are general-purpose, in that they are intended for general trees, not only the syntactic trees as used in standard GP.

\subsubsection{Analysis on fitness distance and tree distance measures}

In Figs. 3, 4 and 5 we present the most representative results on the distribution of fitness distances and three measures (presented in the previous paragraphs) of genotypic distances between individuals which are separated in genetic space by a single mutation. To save space we present only a small selection of results from the various problems and encodings.

Consider first the Artificial Ant problem. Figure 3 shows the fitness and genotypic distance distributions created by the various operators for the $F_{A 4}$ encoding. Although all mutation operators can create very large outliers in fitness distance, it is clear that the normal range of fitness distances created by the Hoist, Size-Fair, and Size-Fair Range mutations is larger than that of the other operators, One-Point, Subtree, and Permutation. A similar pattern holds in the three measures of genetic distance in the same figure. If anything, the difference between operators is stronger in genetic distance than in fitness distance: this shows that although genotypic step-size is a factor in the fitness distance distributions summarised by the three definitions of locality, it is not the only factor. The discrepancy between genotypic step-size distributions and fitness distance distributions is caused by the behaviour of the genotype-fitness mapping.

Similar remarks apply to the Symbolic Regression problem, as illustrated in Fig. 4, where we consider only the $F_{S 6}$ encoding. Again we see relatively larger values for fitness distance, created by the Hoist, Size-Fair, and Size-Fair Range mutations, being reflected in the three genetic distance measures. Note that Subtree mutation is also shown here to also create larger distances than Permutation, and again this is partly reflected in the genetic distances. However, again, genetic distances are not perfect reflections of fitness distances. Genotypic step-size only partially explains the distribution of fitness distance. The discrepancy between genotypic step-size and fitness distance is caused by the behaviour of the genotypefitness mapping. 

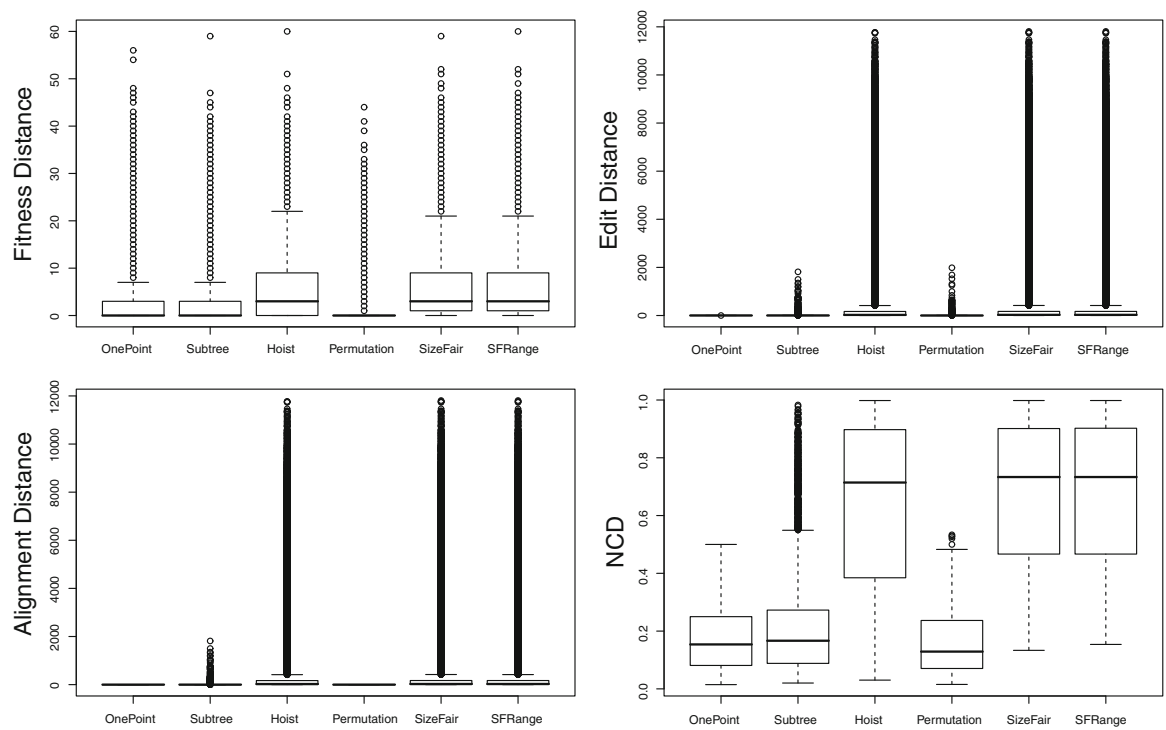

Fig. 3 Fitness distance (top left) and the three distances used in our studies: edit, tree-alignment and normalised compression distance shown in the top right, bottom left and bottom right, respectively. Problem used: Artificial Ant using $F_{A 4}=\{I F, P R O G 2$, PROG3, PROG4 $\}$

Contrast this finally with Fig. 5, again showing the Symbolic Regression problem, this time with the $F_{S 4}$ encoding. Similar patterns in the distances created by the mutations are seen again. The difference is that often much larger outliers are created with the $F_{S 4}$ encoding than $F_{S 6}$. This is notable in both fitness distances and genetic distances. It may that the greater likelihood of mutations involving the discontinuous protected division operator causes this effect.

\section{Conclusions}

It has been argued that locality is a key element in performing effective evolutionary search of landscapes [53]. According to Rothlauf [53], a representation that has high locality is necessary for efficient evolutionary search. The opposite is true when a representation has low locality. To avoid confusion on these terms, we have referred them as locality and non-locality, respectively.

In this work, we have extended the original genotype-phenotype definition of locality (formally introduced and explained in Sect. 2) to the genotype-fitness mapping, considering three different scenarios of fitness neighbourhood.

For this purpose, we have used five problems that include discrete- and continuous-valued ones (both necessary for the understanding of locality in EC). We used three different encodings (two of them induced by the use of alternative function sets and the other by modifying slightly the GP encoding as explained in Sect. 4) for each of theses problems and analysed the locality present under each encoding. 

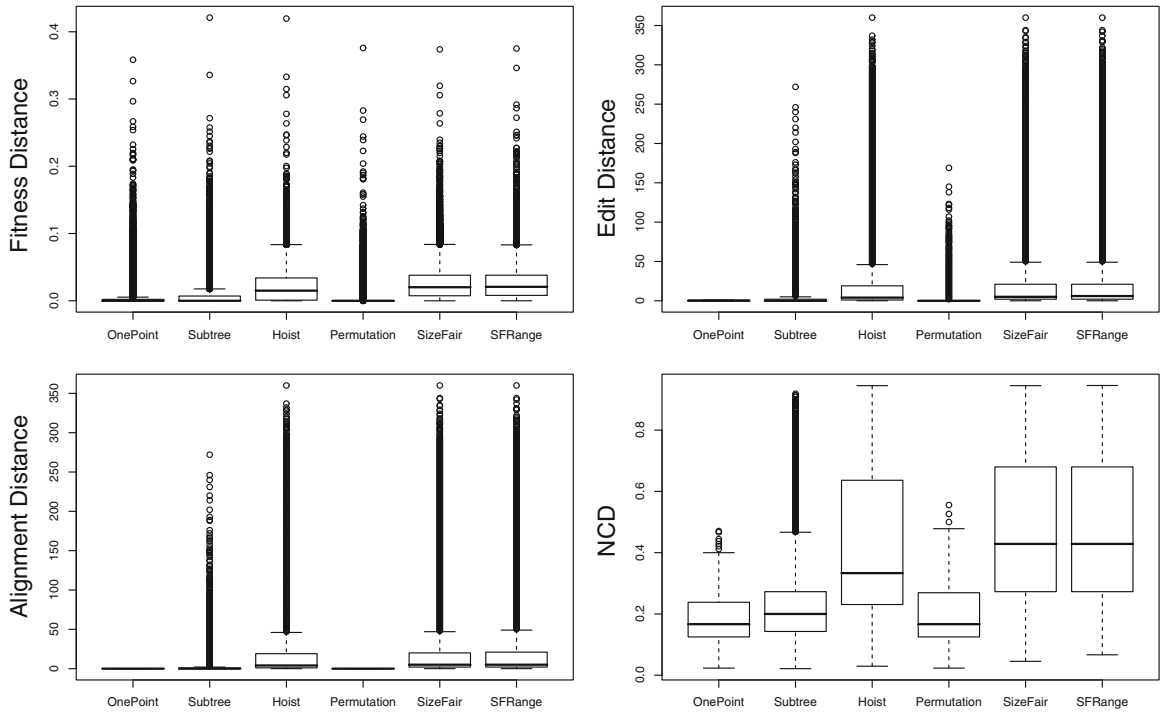

Fig. 4 Fitness distance (top left) and the three distances used in our studies: edit, tree-alignment and normalised compression distance shown in the top right, bottom left and bottom right, respectively. Problem used: symbolic regression $F_{1}$ using $F_{S 6}=\{+,-, *, \%$, Sin, Cos $\}$
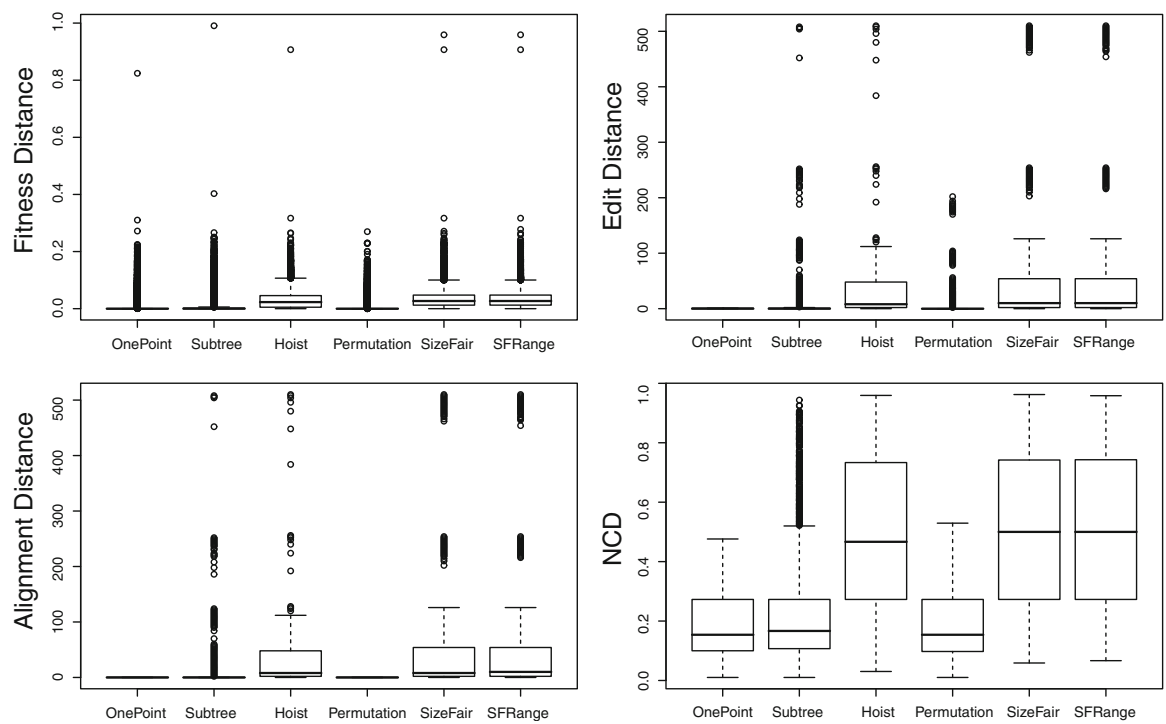

Fig. 5 Fitness distance (top left) and the three distances used in our studies: edit, tree-alignment and normalised compression distance shown in the top right, bottom left and bottom right, respectively. Problem used: symbolic regression $F_{1}$ using $F_{S 4}=\{+,-, *, \%\}$

From the five problems that we have analysed, and using the three different definitions of neighbourhood denoted by $\operatorname{Def}_{0}$, Def $f_{1}$ and $\operatorname{Def}_{2}$ (see Sects. 2.1 and 2.2), we have seen that the correct prediction was obtained more often when 
neighbourhood was defined with Def $_{1}$ which correspond to the definition given by Rothlauf in his original genotype-phenotype mapping studies using bitstrings [53].

To test these quantitative measures of locality, we performed 100 independent runs for all three different encodings, 6 different operators, and three different combinations of population sizes and number of generations. In almost all of them, we have confirmed the prediction made by locality. It is, however, fair to say that none of the locality definitions presented in Sect. 2 was able to predict performance all the time (e.g., different mutation operators, population sizes, generations and more). One can argue that for any performance prediction approach, there is always a counter example, as shown in various works (e.g., [30, 67]).

\section{Future work}

We have made an effort to shed some light on locality in Genetic Programming. For this purpose, we started our analysis by using by using only mutations. This has helped us to better understand how locality influences evolutionary search. A natural step to take from here is by considering the use of crossover.

Also, to compare the predictions made by locality against performance, we used one of the most popular sampling methods by considering the use of the ramped half-and-half method. As mentioned previously, this approach has some limitations, so it would be interesting to explore other sampling methods (some of them were detailed in Sect. 4).

Acknowledgments This research is based upon works supported by Science Foundation Ireland under Grant No. 08/IN.1/I1868 and by the Irish Research Council for Science, Engineering and Technology under the Empower scheme. The authors would like to thank the anonymous reviewers for their valuable comments. Leonardo Vanneschi is particularly thanked for his helpful suggestions and for his encouragement to further continue developing this research.

\section{Appendix}

Tables 9, 11, 13, 15 and 17 show the results on locality and Tables 10, 12, 14, 16 and 18 show the performance (measured in terms of average of the best fitness

Table 9 Locality on the Even-3-Parity Problem using three function sets $\left(F_{E 3}=\{A N D, O R, N O T\}\right.$, $F_{E 4}=\{A N D, O R, N A N D, N O R\}$ and $\left.F_{E 3^{*}}=\{A N D, O R, N O T 2\}\right)$, six mutations, and three locality definitions. Lower is better

\begin{tabular}{|c|c|c|c|c|c|c|c|c|c|}
\hline \multirow[t]{2}{*}{ Mutation operators } & \multicolumn{3}{|c|}{$f d_{\min }=0$} & \multicolumn{3}{|c|}{$f d_{\min }=1$} & \multicolumn{3}{|c|}{ Cond. $\left(f d_{\min }=1\right)$} \\
\hline & $F_{E 3}$ & $F_{E 4}$ & $F_{E 3^{*}}$ & $F_{E 3}$ & $F_{E 4}$ & $F_{E 3^{*}}$ & $F_{E 3}$ & $F_{E 4}$ & $F_{E 3^{*}}$ \\
\hline One $\mathrm{p}$ & 0.1125 & 0.1727 & 0.1059 & 0.9057 & 0.8699 & 0.9145 & 0.0091 & 0.0213 & 0.0102 \\
\hline Subtree & 0.1599 & 0.1 & 0.1026 & 0.8562 & 0.8541 & 0.9064 & 0.0081 & 0.0089 & 0.0045 \\
\hline Permutation & 0 & 0 & 0.0154 & 1 & 1 & 0.9859 & 0 & 0 & 0.0006 \\
\hline Hoist & 0.1902 & 0.2497 & 0.1932 & 0.8376 & 0.7936 & 0.8304 & 0.0139 & 0.0217 & 0.0118 \\
\hline Size fair & 0.2046 & 0.2710 & 0.2063 & 0.8173 & 0.7707 & 0.8150 & 0.0109 & 0.0209 & 0.0106 \\
\hline Size fair* & 0.1993 & 0.2641 & 0.2017 & 0.8207 & 0.7742 & 0.8179 & 0.0100 & 0.0191 & 0.0098 \\
\hline
\end{tabular}




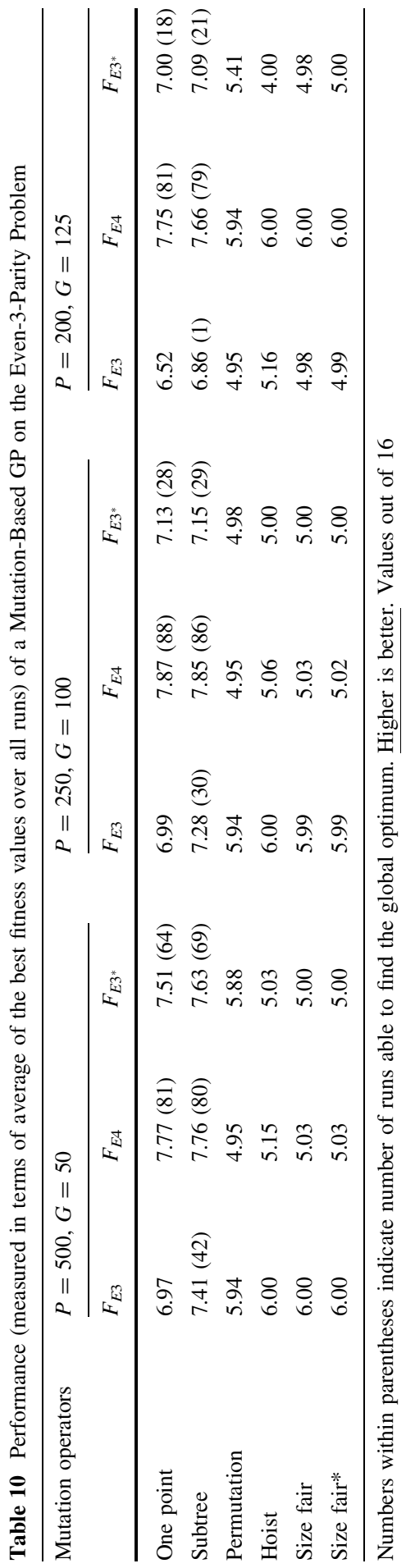


Table 11 Locality on the Even-4-Parity Problem using three function sets $\left(F_{E 3}=\{A N D, O R\right.$, $N O T\}, F_{E 4}=\{A N D, O R, N A N D, N O R\}$ and $\left.F_{E 3^{*}}=\{A N D, O R, N O T 2\}\right)$, six mutations, and three locality definitions. Lower is better

\begin{tabular}{|c|c|c|c|c|c|c|c|c|c|}
\hline \multirow[t]{2}{*}{ Mutation operators } & \multicolumn{3}{|c|}{$f d_{\min }=0$} & \multicolumn{3}{|c|}{$f d_{\min }=1$} & \multicolumn{3}{|c|}{ Cond. $\left(f d_{\min }=1\right)$} \\
\hline & $F_{E 3}$ & $F_{E 4}$ & $F_{E 3^{*}}$ & $F_{E 3}$ & $F_{E 4}$ & $F_{E 3^{*}}$ & $F_{E 3}$ & $F_{E 4}$ & $F_{E 3^{*}}$ \\
\hline One point & 0.8623 & 0.1308 & 0.0629 & 0.9297 & 0.9027 & 0.9505 & 0.0080 & 0.0167 & 0.0067 \\
\hline Subtree & 0.1307 & 0.1326 & 0.0664 & 0.8809 & 0.8816 & 0.9388 & 0.0058 & 0.0071 & 0.0026 \\
\hline Permu & 0 & 0 & 0.0109 & 1 & 1 & 0.9900 & 0 & 0 & 0.0005 \\
\hline Hoist & 0.1526 & 0.2121 & 0.1365 & 0.8686 & 0.8304 & 0.8801 & 0.0106 & 0.0213 & 0.0083 \\
\hline Size fair & 0.1618 & 0.2216 & 0.1413 & 0.8554 & 0.8179 & 0.8737 & 0.0086 & 0.0197 & 0.0075 \\
\hline Size fair* & 0.1590 & 0.2166 & 0.1396 & 0.8579 & 0.8216 & 0.8748 & 0.0084 & 0.0191 & 0.0072 \\
\hline
\end{tabular}

Table 12 Performance (measured in terms of average of the best fitness values over all runs) of a mutation-based GP on the Even-4-Parity problem

\begin{tabular}{|c|c|c|c|c|c|c|c|c|c|}
\hline \multirow[t]{2}{*}{ Mutation operators } & \multicolumn{3}{|c|}{$P=500, G=50$} & \multicolumn{3}{|c|}{$P=250, G=100$} & \multicolumn{3}{|c|}{$P=200, G=125$} \\
\hline & $F_{E 3}$ & $F_{E 4}$ & $F_{E 3^{*}}$ & $F_{E 3}$ & $F_{E 4}$ & $F_{E 3^{*}}$ & $F_{E 3}$ & $F_{E 4}$ & $F_{E 3^{*}}$ \\
\hline One point & 11.89 & 14.35 (29) & 12.16 & 11.35 & $13.66(25)$ & 11.66 & 10.88 & 13.40 & 11.29 \\
\hline Subtree & 12.43 & $14.12(15)$ & 12.35 & 12.59 & $13.18(11)$ & 11.65 & 12.23 & $13.28(9)$ & 11.03 \\
\hline Perm & 9.90 & 8.91 & 9.89 & 9.90 & 9.90 & 8.91 & 8.91 & 8.91 & 8.91 \\
\hline Hoist & 9.90 & 8.95 & 8.91 & 9.89 & 9.89 & 8.91 & 8.91 & 8.91 & 8.91 \\
\hline Size fair & 9.90 & 8.95 & 8.92 & 9.89 & 9.89 & 8.91 & 8.90 & 8.90 & 8.90 \\
\hline Size fair* & 9.90 & 8.93 & 8.91 & 9.88 & 9.89 & 8.91 & 8.90 & 8.89 & 8.90 \\
\hline
\end{tabular}

Numbers within parentheses indicate number of runs able to find the global optimum. Higher is better. Values out of 32

Table 13 Locality on the Artificial Ant Problem using three function sets $\left(F_{A 3}=\{I F, P R O G 2\right.$, $P R O G 3\}, F_{A 4}=\{I F, P R O G 2, P R O G 3, P R O G 4\}$ and $\left.F_{A 3^{*}}=\{I F 3, P R O G 23, P R O G 3\}\right)$, six mutations, and three locality definitions. Lower is better

\begin{tabular}{|c|c|c|c|c|c|c|c|c|c|}
\hline \multirow[t]{2}{*}{ Mutation operators } & \multicolumn{3}{|c|}{$f d_{\min }=0$} & \multicolumn{3}{|c|}{$f d_{\min }=1$} & \multicolumn{3}{|c|}{ Cond. $\left(f d_{\min }=1\right)$} \\
\hline & $F_{A 3}$ & $F_{A 4}$ & $F_{A 3^{*}}$ & $F_{A 3}$ & $F_{A 4}$ & $F_{A 3^{*}}$ & $F_{A 3}$ & $F_{A 4}$ & $F_{A 3^{*}}$ \\
\hline One $\mathrm{p}$ & 1.8209 & 2.0572 & 1.0045 & 2.0674 & 2.2104 & 1.5639 & 1.4442 & 1.6338 & 0.7841 \\
\hline Subtree & 2.0631 & 2.2584 & 0.9619 & 2.1980 & 2.3319 & 1.5299 & 1.6306 & 1.7951 & 0.7459 \\
\hline Permutation & 1.1468 & 1.2881 & 0.9440 & & & 1.5020 & 0.8851 & 0.9844 & 0.7229 \\
\hline Hoist & 4.0950 & 4.7628 & 4.2617 & 3.8720 & 4.7628 & 3.9515 & 3.4835 & 4.4549 & 3.6066 \\
\hline Size fair & 4.5438 & 5.5208 & 4.5167 & 4.0931 & 4.9515 & 4.0606 & 3.8185 & 4.7361 & 3.7887 \\
\hline Size fair* & 4.5350 & 5.5323 & 4.5051 & 4.0869 & 4.9645 & 4.0548 & 3.8109 & 4.7486 & 3.7799 \\
\hline
\end{tabular}




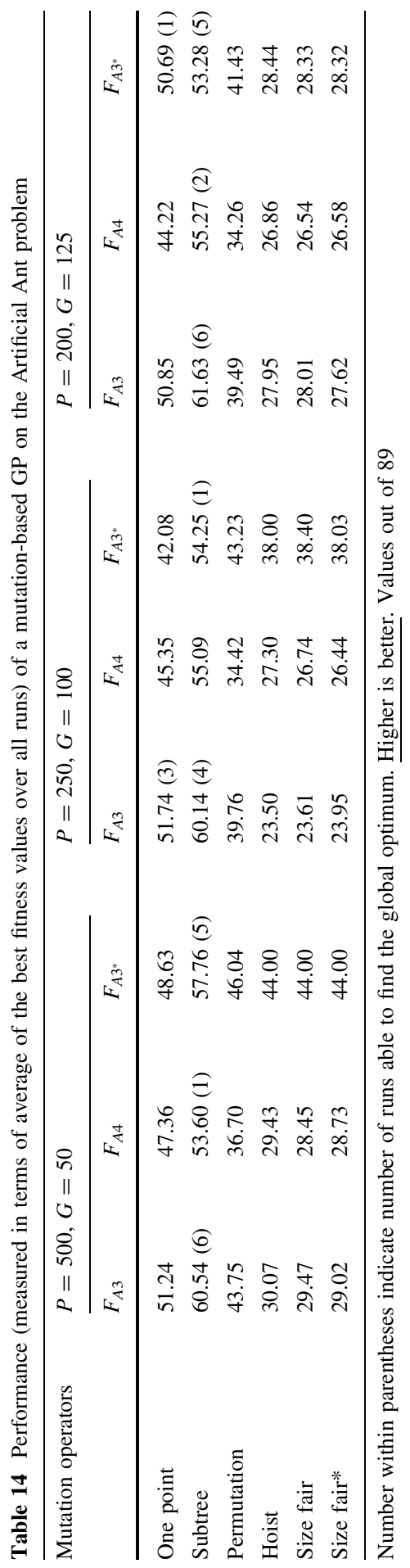




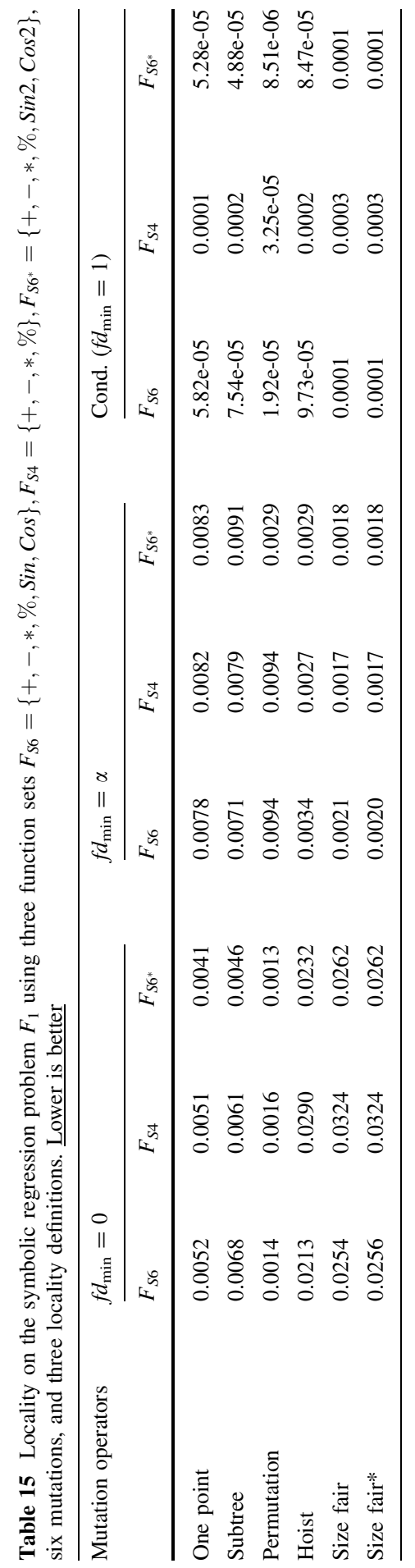




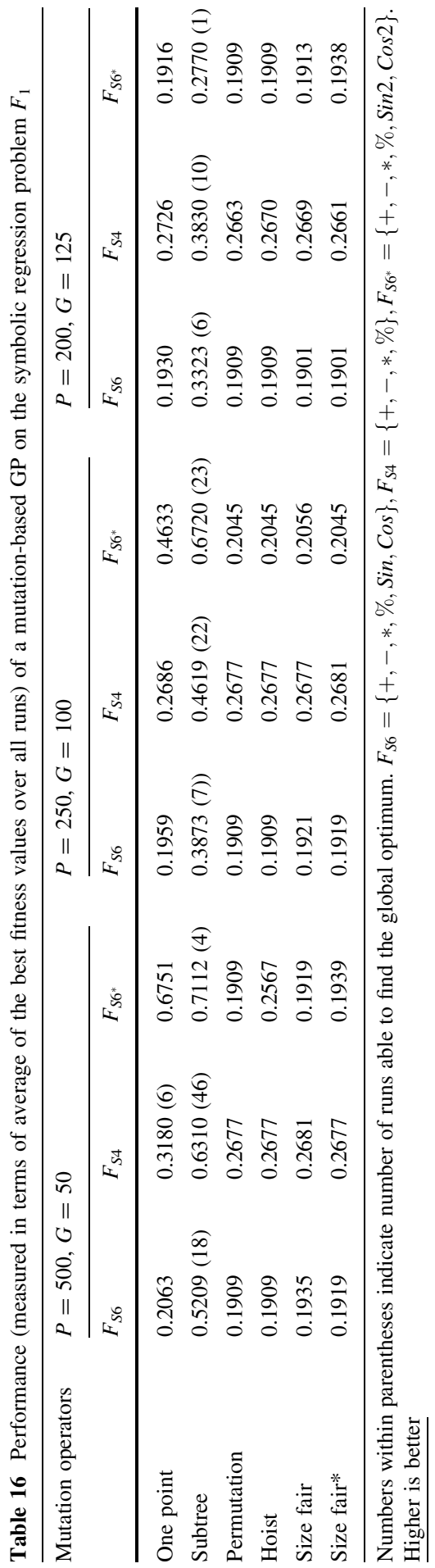




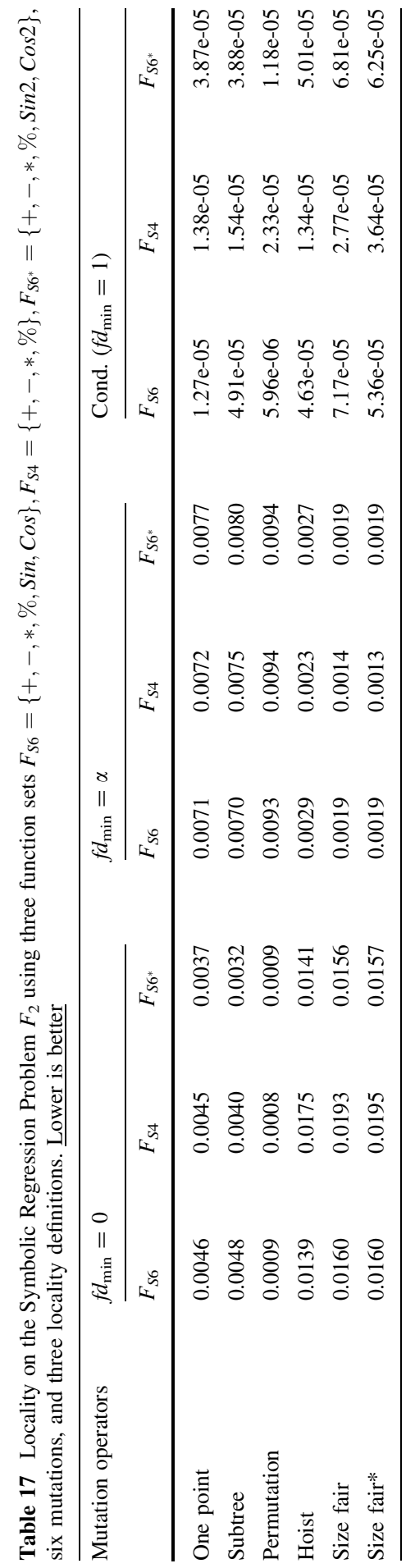




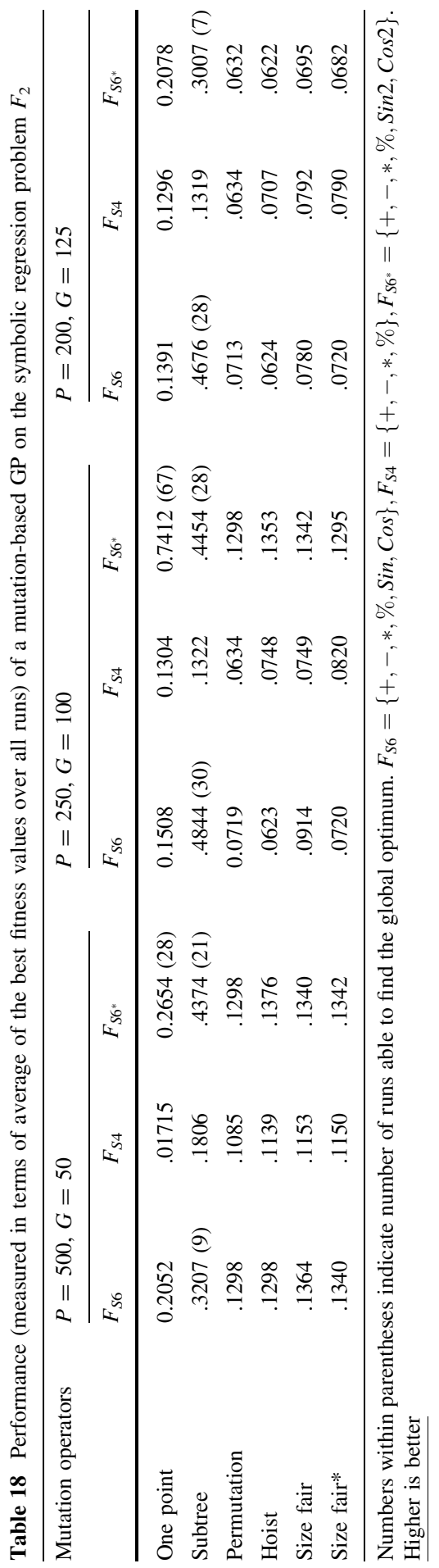


values over all runs) for the Even-3, Even-4, Artificial Ant and two Symbolic Regression problems $\left(F_{1}\right.$ and $\left.F_{2}\right)$, respectively.

\section{References}

1. L. Altenberg, Fitness Distance Correlation Analysis: An Instructive Counterexample. in Proceedings of the Seventh International Conference on Genetic Algorithms, ed. by T. Back (Morgan Kaufmann, 1997), pp 57-64, San Francisco, CA, USA

2. H. Beyer, H. Schwefel, Evolution strategies - a comprehensive introduction. Nat. Comput. 1(1), 3-52 (2002)

3. M. Brameier, W. Banzhaf, Linear Genetic Programming. (Springer, New York, 2006)

4. R. Cilibrasi, P.M.B. Vitanyi, Clustering by compression. IEEE Trans. Inf. Theory 51(4), 1523-1545 (2005)

5. M. Clergue, P. Collard, GA-Hard Functions Built by Combination of Trap Functions. In: D.B. Fogel, M.A. El-Sharkawi, X. Yao, G. Greenwood, H. Iba, P. Marrow, M. Schackleton (eds) CEC 2002: Proceedings of the 2002 Congress on Evolutionary Computation, (IEEE Press, New York, 2002) pp. 249-254.

6. M. Clergue, P. Collard, M. Tomassini, L. Vanneschi, in Proceedings of the Genetic and Evolutionary Computation Conference, GECCO 2002, eds. by W.B. Langdon, E. Cantú-Paz, K.E. Mathias, R. Roy, D. Davis, R. Poli, K. Balakrishnan, V. Honavar, G. Rudolph, J. Wegener, L. Bull, M.A. Potter, A.C. Schultz, J.F. Miller, E.K. Burke, N. Jonoska. Fitness Distance Correlation and Problem Difficulty for Genetic Programming (Morgan Kaufmann Publishers, New York, 2002), pp. 724-732

7. I. De Falco, A. Iazzetta, E. Tarantino, A. Della Cioppa, G. Trautteur, in Proceedings of the Genetic and Evolutionary Computation Conference (GECCO-2000). A Kolmogorov Complexity Based Genetic Programming Tool for String Compression (2000)

8. P. D'haeseleer, J. Bluming, Effects of Locality in Individual and Population Evolution. In: K.E. Kinnear (eds) Advances in Genetic Programming, (MIT Press, Cambridge, 1994) pp. 177-198.

9. A. Ekárt, S.Z. Németh, in EuroGP, number 1802 in Lecture Notes in Computer Science. A metric for genetic programs and fitness sharing (Springer, 2000), pp. 259-270

10. D.B. Fogel, A. Ghozeil, Using Fitness Distributions to Design More Efficient Evolutionary Computations (1996)

11. E. Galván-López, An Analysis of the Effects of Neutrality on Problem Hardness for Evolutionary Algorithms. PhD thesis, School of Computer Science and Electronic Engineering, University of Essex, United Kingdom (2009)

12. E. Galván-López, S. Dignum, R. Poli, The Effects of Constant Neutrality on Performance and Problem Hardness in GP. in EuroGP 2008 - 11th European Conference on Genetic Programming, vol. 4971 of $L N C S$, ed. by M. O’Neill, L. Vanneschi, S. Gustafson, A.I.E. Alcazar, I.D. Falco, A.D. Cioppa, E. Tarantino (Springer, 26-28 Mar. 2008), pp. 312-324, Napoli, Italy

13. E. Galván-Lopéz, J. McDermott, M. O’Neill, A. Brabazon, in CEC 2010: Proceedings of the 12th Annual Congress on Evolutionary Computation. Defining locality in genetic programming to predict performance, Barcelona, Spain (IEEC Press, July 2010)

14. E. Galván-Lopéz, J. McDermott, M. O’Neill, A. Brabazon, in GECCO 2010: Proceedings of the 12th Annual Conference on Genetic and Evolutionary Computation. Towards an understanding of locality in genetic programming. (ACM Press, Portland, July 2010)

15. E. Galván-López, M. O’Neill, A. Brabazon, in Artificial Intelligence, 2009. MICAI 2009. Eighth Mexican International Conference on. Towards Understanding the Effects of Locality in gp (2009), pp. $9-14$

16. E. Galván-López, R. Poli, in Parallel Problem Solving from Nature (PPSN IX). 9th International Conference, vol. 4193 of LNCS, ed. by T.P. Runarsson, H.-G. Beyer, E. Burke, J.J. Merelo-Guervós, L.D. Whitley, X. Yao. Some Steps Towards Understanding How Neutrality Affects Evolutionary Search (Springer, 9-13 Sept. 2006), pp. 778-787 (Reykjavik, Iceland)

17. E. Galván-López, R. Poli, in MICAI, vol. 5845 of Lecture Notes in Computer Science, ed. by A.H. Aguirre, R.M. Borja, C.A.R. Garcia. An Empirical Investigation of How Degree Neutrality Affects gp Search (Springer, 2009), pp. 728-739 
18. E. Galván López, R. Poli, C.A. Coello Coello, in Genetic Programming 7th European Conference, EuroGP 2004, Proceedings, vol. 3003 of LNCS, ed. by M. Keijzer, U.-M. O'Reilly, S. Lucas, E. Costa, T. Soule. Reusing Code in Genetic Programming (Springer, 5-7 Apr. 2004), pp. 359-368 (Coimbra, Portugal)

19. E. Galván-López, R. Poli, A. Kattan, M. O’Neill, A. Brabazon, Neutrality in evolutionary algorithms ... what do we know? Evol. Syst. (2011)

20. D.E. Goldberg, Construction of high-order deceptive functions using low-order walsh coefficients. Ann. Math. Artif. Intell. 5(1), 35-47 (1992)

21. D.E. Goldberg, K. Deb, J. Horn, in PPSN II: Proceedings of the 2nd International Conference on Parallel Problem Solving from Nature, ed. by R. Männer, B. Manderick. Massive Multimodality, Deception, and Genetic Algorithms (Elsevier, Amsterdam, 1992), pp. 37-48

22. F.J. Gomez, in Proceedings of the 11th Annual conference on Genetic and evolutionary computation. Sustaining Diversity Using Behavioral Information Distance (ACM, Montréal, 2009), pp. 113-120

23. J. Gottlieb, B.A. Julstrom, G.R. Raidl, F. Rothlauf, in Proceedings of the Genetic and Evolutionary Computation Conference (GECCO 2001), ed. by S. Spector, E. Wu, B. Voigt, Gen, Sen, Dorigo, Pezeshk, Garzon, Burke. Prufer numbers: A poor representation of spanning trees for evolutionary search (Morgan Kaufmann, 2001), pp. 343-350

24. J. Gottlieb, G.R. Raidl, in Proceedings of the Genetic and Evolutionary Computation Conference 2000. The Effects of Locality on the Dynamics of Decoder-Based Evolutionary Search

25. J. Gottlieb, G.R. Raidl, in AE '99: Selected Papers from the 4th European Conference on Artificial Evolution. Characterizing Locality in Decoder-Based EAs for the Multidimensional Knapsack Problem (Springer, London, 2000), pp. 38-52

26. S. Gustafson, L. Vanneschi, Crossover-based tree distance in genetic programming. IEEE Trans. Evol. Comput. 12(4), 506-524 (2008)

27. J.H. Holland, Adaptation in Natural and Artificial Systems. (University of Michigan Press, Ann Arbor, 1975)

28. C. Igel, K. Chellapilla, Investigating the Influence of Depth and Degree of Genotypic Change on Fitness in Genetic Programming (1999)

29. T. Jiang, L. Wang, K. Zhang, Alignment of trees - an alternative to tree edit. Theor. Comput. Sci. 143(1), 137-148 (1995)

30. T. Jones. Evolutionary Algorithms, Fitness Landscapes and Search. PhD thesis, University of New Mexico, Albuquerque (1995)

31. T. Jones, S. Forrest, in Proceedings of the 6th International Conference on Genetic Algorithms, ed. by L.J. Eshelman. Fitness Distance Correlation as a Measure of Problem Difficulty for Genetic Algorithms (Morgan Kaufmann Publishers, San Francisco, 1995), pp. 184-192

32. K.E. Kinnear, Jr., in Proceedings of the 1994 IEEE World Conference on Computational Intelligence, vol. 1. Fitness Landscapes and Difficulty in Genetic Programming (IEEE Press, Orlando, 27-29 June 1994) pp. 142-147

33. J.R. Koza, Genetic Programming: On the Programming of Computers by Means of Natural Selection. (The MIT Press, Cambridge, 1992)

34. W. Langdon, R. Poli, Why Ants are Hard. In: J.R. Koza (eds) Proceedings of the Third Annual Conference on Genetic Programming, (Morgan Kaufmann, Madison, 1998) pp. 193-201.

35. W.B. Langdon, in 1998 IEEE International Conference on Evolutionary Computation. The Evolution of Size in Variable Length Representations (IEEE Press, 1998), pp. 633-638

36. W.B. Langdon, R. Poli, Foundations of Genetic Programming. (Springer, Berlin, 2002)

37. P.K. Lehre, P.C. Haddow, Phenotypic complexity and local variations in neutral degree. BioSystems 87(2-3), 233-242 (2006)

38. M. Li, P. Vitanyi, An Introduction to Kolmogorov Complexity and its Applications. (Springer, Berlin, 1997)

39. B. Manderick, de M.K. Weger, P. Spiessens, The Genetic Algorithm and the Structure of the Fitness Landscape. In: R.K. Belew, L.B. Booker (eds) ICGA, (Morgan Kaufmann, Los Altos, 1991) pp. 143-150.

40. J.F. Miller, P. Thomson, in EuroGP. Cartesian Genetic Programming (Springer, 2000), pp. 121-132

41. B. Naudts, L. Kallel, A comparison of predictive measures of problem difficulty in evolutionary algorithms. IEEE Trans. Evol. Comput. 4(1), 1-15 (2000)

42. P. Nordin, Evolutionary Program Induction of Binary Machine Code and its Applications. PhD thesis, der Universitat Dortmund am Fachereich Informatik (1997) 
43. U.-M. O'Reilly, in IEEE International Conference on Systems, Man, and Cybernetics: Computational Cybernetics and Simulation, vol. 5. Using a Distance Metric on Genetic Programs to Understand Genetic Operators (1997)

44. R. Poli, E. Galván-López, in Foundations of Genetic Algorithms IX, Lecture Notes in Computer Science, ed. by C.R. Stephens, M. Toussaint, D. Whitley, P. Stadler. On The Effects of Bit-Wise Neutrality on Fitness Distance Correlation, Phenotypic Mutation Rates and Problem Hardness (Springer, Mexico city, 8-11 Jan. 2007), pp. 138-164

45. R. Poli, E. Galván-López, The Effects of Constant and Bit-Wise Neutrality on Hardness, Fitness Distance Correlation and Phenotypic Mutation Rataes. IEEE Trans. Evol. Comput. (2011)

46. R. Poli, W.B. Langdon, N.F. McPhee, A field guide to genetic programming. Published via http://lulu.com and freely available at http://www.gp-field-guide.org.uk, 2008. (With contributions by J. R. Koza)

47. R. Poli, L. Vanneschi, in Proceedings of the 9th annual conference on Genetic and evolutionary computation, GECCO '07. Fitness-Proportional Negative Slope Coefficient as a Hardness Measure for Genetic Algorithms (ACM, New York, 2007), pp. 1335-1342

48. B. Punch, D. Zongker, E. Godman, in Advances in Genetic Programming 2, ed. by P. Angeline, K. Kinnear. The Royal Tree Problem, A Benchmark for Single and Multi-population Genetic Programming (The MIT Press, Cambridge, 1996), pp. 299-316

49. R.J. Quick, V.J. Rayward-Smith, G.D. Smith, in Proceedings of the 5th International Conference on Parallel Problem Solving from Nature. Fitness Distance Correlation and Ridge Functions (Springer, London, 1998), pp. 77-86

50. I. Rechenberg, Evolutionsstrategie 94, volume 1 of Werkstatt Bionik und Evolutionstechnik. (Frommann-Holzboog, Stuttgart, 1994)

51. S. Ronald, Robust Encodings in Genetic Algorithms. In: Z. Michalewicz, K. Deb, M. Schmidt, T. Stidsen (eds) Evolutionary Algorithms in Engineering Applications, (Springer, Berlin, 1997) pp. 29-44.

52. F. Rothlauf, Representations for Genetic and Evolutionary Algorithms, 2nd edn. (Physica, Berlin, 2006)

53. F. Rothlauf, D. Goldberg, Redundant representations in evolutionary algorithms. Evol. Comput. 11(4), 381-415 (2003)

54. F. Rothlauf, D.E. Goldberg, Pruefer numbers and genetic algorithms: A lesson how the low locality of an encoding can harm the performance of GAs. Technical Report 3/2000, Bayreuth (2000)

55. F. Rothlauf, E. Goldberg, David, Tree network design with genetic algorithms—an investigation in the locality of the pruefernumber encoding. Technical Report 6/1999, Bayreuth (1999)

56. F. Rothlauf, M. Oetzel, in Proceedings of the 9th European Conference on Genetic Programming, vol. 3905 of Lecture Notes in Computer Science, ed. by P. Collet, M. Tomassini, M. Ebner, S. Gustafson, A. Ekárt. On the Locality of Grammatical Evolution (Springer, Budapest, 10-12 Apr. 2006), pp. 320-330

57. D. Shasha, K. Zhang, in SPAA '89: Proceedings of the First Annual ACM Symposium on Parallel Algorithms and Architectures. Fast Parallel Algorithms for the Unit Cost Editing Distance Between Trees (ACM, New York, 1989), pp. 117-126

58. P.F. Stadler, C.R. Stephens, Landscapes and effective fitness. Comments Theori. Biol. 8, 389-431 (2002)

59. M. Tacker, P.F. Stadler, E.G. Bornberg-Bauer, I.L. Hofacker, P. Schuster, Algorithm indepedent properties of RNA secondary structure predictions. Eur. Biophys. J. 25(2), 115-130 (1996)

60. M. Tomassini, L. Vanneschi, P. Collard, M. Clergue, A study of fitness distance correlation as a difficulty measure in genetic programming. Evol. Comput. 13(2), 213-239 (2005)

61. M. Toussaint, C. Igel, in Proceedings of the IEEE Congress on Evolutionary Computation (CEC 2002). Neutrality: A Necessity for Self-Adaptation (2002), pp. 1354-1359

62. L. Vanneschi, Theory and Practice for Efficient Genetic Programming. PhD thesis, Faculty of Science, University of Lausanne, Switzerland (2004)

63. L. Vanneschi, in Genetic Programming Theory and Practive V, chap. 7, ed. by R. et al. Investigating Problem Hardness of Real Life Applications (Springer, US, 2007), pp. 107-124

64. L. Vanneschi, M. Clergue, P. Collard, M. Tomassini, S. Verel, in EuroGP, LNCS. Fitness Clouds and Problem Hardness in Genetic Programming (Springer, Berlin, 2004), pp. 690-701

65. L. Vanneschi, M. Tomassini, P. Collard, M. Clergue, in EuroGP, Lecture notes in computer science. Fitness Distance Correlation in Structural Mutation Genetic Programming (Springer, Berlin, 2003), pp. 455-464 
66. L. Vanneschi, M. Tomassini, P. Collard, S. Verel, Y. Pirola, G. Mauri, in Proceedings of EuroGP 2007, vol. 4445 of LNCS. A comprehensive View of Fitness Landscapes with Neutrality and Fitness Clouds (Springer, Berlin, 2007), pp. 241-250

67. L. Vanneschi, A. Valsecchi, R. Poli, in GECCO '09: Proceedings of the 11th Annual Conference on Genetic and Evolutionary Computation. Limitations of the Fitness-Proportional Negative Slope Coefficient as a Difficulty Measure (ACM, New York, 2009), pp. 1877-1878

68. E. Weinberger, Correlated and uncorrelated fitness landscapes and how to tell the difference. Biol. Cybern. 63(5), 325-336 (1990)

69. S. Wright, in Proceedings of the Sixth International Congress on Genetics, vol. 1, ed. by D.F. Jones. The Roles of Mutation, Inbreeding, Crossbreeding and Selection in Evolution (1932), pp. 356-366 